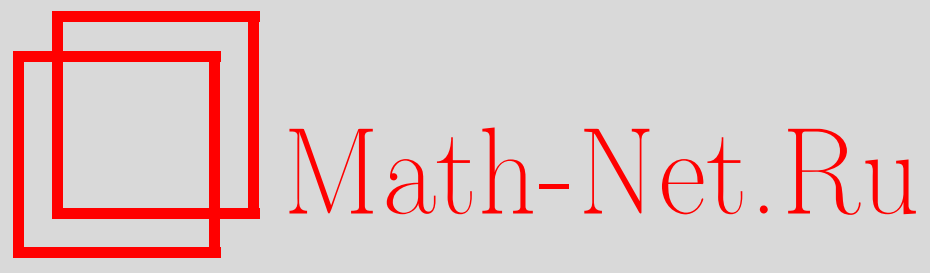

В. А. Ватутин, В. А. Топчий, Предельная теорема для критических каталитических ветвящихся случайных блужданий, Теория вероятн. и ее примен., 2004, том 49, выпуск 3, 461-484

DOI: https://doi.org/10.4213/tvp203

Использование Общероссийского математического портала MathNet.Ru подразумевает, что вы прочитали и согласны с пользовательским соглашением

http://www . mathnet.ru/rus/agreement

Параметры загрузки:

IP : 52.87 .193 .239

26 апреля 2023 г., 10:28:40

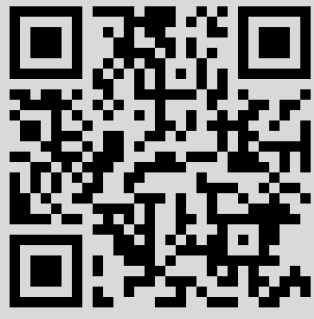




\title{
ПРЕДЕЛЬНАЯ ТЕОРЕМА ДЛЯ \\ КРИТИЧЕСКИХ КАТАЛИТИЧЕСКИХ ВЕТВЯЩИХСЯ СЛУЧАЙНЫХ БЛУЖДАНИЙ ${ }^{1)}$
}

\begin{abstract}
Рассматривается ветвящееся случайное блуждание с непрерывным временем по решетке $\mathbf{Z}$, в котором частицы могут гибнуть и производить потомство, лишь находясь в нуле.

В предположении, что базовое марковское случайное блуждание однородно и симметрично, а средняя численность потомства одной частицы равна 1 , описывается асимптотическое поведение при $t \rightarrow \infty$ условного распределения соответствующим образом нормированного двумерного вектора $(\zeta(t), \mu(t))$, где $\zeta(t)$ пи $\mu(t)$ - количество частиц в нуле и вне нуля в момент $t$, при условии $\zeta(t)>0$.
\end{abstract}

Ключевые слова и фразы: критический ветвящийся процесс Беллмана-Харриса с двумя типами частиц, неоднородное ветвящееся случайное блуждание по решетке на прямой, предельные теоремы.

1. Введение. Каталитическим ветвящимся случайным блужданием мы будем называть следуюшую версию ветвящегося случайного блуждания с непрерывным временем по решетке Z. Находящиеся вне нуля частицы блуждают без преврашений по решетке $\mathbf{Z}$ до момента попадания в точку $x=0$. Частицы, попавшие в точку $x=0$, проводят в этой точке случайное время, а затем либо гибнут, производя в нулевой точке потомство, либо совершают мгновенный прыжок в одно из ненулевых состояний. Эволюция новорожденных частиц не зависит от предыстории процесса, а также перемещения и размножения остальных частиц.

* Математический институт им. В. А. Стеклова РАН, ул. Губкина, 8, 119991 Москва, Россия; e-mail: vatutin@mi.ras.ru

** Омский филиал Института математики им. В. Л. Соболева СО РАН, ул. ПІевцова, 13, 644099 Омск Россия; e-mail: topchij@iitam.omsk.net.ru

1) Первый автор поддержан РФФИ (грант № 02-01-00266), фондом Президента РФ (грант НШ-1758.2003.1), программой «Современные проблемы теоретической математики» PAH и INTAS (грант № 03-51-5018); второй автор поддержан РФФИ (грант № 03-01-00045), фондом Президента РФ (грант № НШ-2139.2003.1) и программой 1.1 Отделения математических наук $\mathrm{PAH}$ «Современные проблемы теоретической математики в ИМ CO PAH». 
Дадим более формальное описание интересующей нас модели. Будем предполагать, что перемещение частиц по целочисленной решетке $\mathbf{Z}$ (за исключением тех, которые выходят из нуля, не производя потомства) происходит в соответствии с матрицей интенсивностей переходных ве-

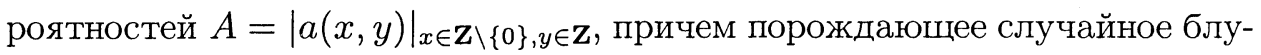
ждание по решетке $\mathbf{Z}$ удовлетворяет следующим условиям. Оно

- однородно: $a(x, y) \stackrel{\text { def }}{=} a(y-x)$ при $x \neq 0$, причем $a(z) \geqslant 0$ при $z \neq 0$ и $a(0)<0$;

- симметрично: $a(-x)=a(x)$;

- неразложимо: из любого состояния за некоторое число шагов можно попасть в любое другое состояние, или, что то же самое, н.о.д. $\{x \neq 0: a(x)>0\}=1$;

- регулярно: $\sum_{x \in \mathbf{Z}} a(x)=0$.

Интенсивности переходов из нуля пропорциональны интенсивностям переходов из других состояний и имеют вид $a(0, x)=-a(x) / a(0)$. Это означает, что вероятности переходов из состояния в состояние для блуждания однородны по пространству и времени, причем время пребывания в состоянии $x=0$ имеет экспоненциальное распределение со средним 1 , тогда как время пребывания в любом другом состоянии $x \neq 0$ имеет экспоненциальное распределение со средним $-1 / a(0)$.

Развитие популяции начинается в момент времени $t=0$ с одной частицы, расположенной в нуле. Начальная частица, так же как и любая другая частица, оказавшаяся в точке нуль позднее, проводит в этой точке случайное время, имеющее экспоненциальное распределение с единичным средним, а затем либо переходит в точку $y \neq 0$ с вероятностью $(1-\alpha) a(0, y)$, либо гибнет с вероятностью $\alpha$, порождая при этом случайное количество потомков $\xi$ в соответствии с производящей функцией

$$
f(s) \stackrel{\text { def }}{=} \mathbf{E} s^{\xi}=\sum_{k=0}^{\infty} f_{k} s^{k} .
$$

Новорожденные частицы появляются в нуле и с момента рождения эволюционируют независимо друг от друга и от остальных частиц. Их превращения стохастически эквивалентны блужданиям с возможной гибелью и ветвлением в нуле для начальной частицы (отметим, что частицы могут делиться, не выходя из нуля, или при любом из возвращений в него).

Пусть $\zeta(t)$ и $\mu(t)$ - количество частиц в нуле и вне нуля в момент $t$ соответственно. Целью работы является описание асимптотического поведения при $t \rightarrow \infty$ условного распределения подходящим образом нормированного двумерного вектора $(\zeta(t), \mu(t))$ при условии $\zeta(t)>0$.

Отметим, что изучение ветвящихся процессов в каталитической среде было начато в работах [4]-[7], где (в несколько более общей ситуации блужданий по решетке $\mathbf{Z}^{d}$ ) выведены интегральные уравнения 
для производящих функций случайных величин $\zeta(t, x)$ - частиц, находящихся в момент $t$ в точке $x \in \mathbf{Z}^{d}$, и величины $\eta(t)$ - размера популяции в момент $t$, а также исследовано асимптотическое поведение моментов указанных величин. Настоящая работа является продолжением статей [1] и [2], в которых для случая $d=1$ была найдена асимптотика вероятностей $\mathbf{P}\{\eta(t)>0\}$ и $\mathbf{P}\{\zeta(t, 0)>0\}$ и доказаны одномерные условные предельные теоремы для величин $\zeta(t)=\zeta(t, 0)$ и $\eta(t)$ при условиях $\zeta(t)>0$ и $\eta(t)>0$ соответственно.

Заметим, что ветвящиеся случайные блуждания в каталитической среде могут быть использованы в качестве аппроксимаций каталитических суперпрочессов (см., например, [8] и [9]). Описанию аппроксимаций такого рода посвящена отдельная статья (см. [13]).

Всюду далее предполагается, что выполнены следующие условия:

$$
b^{2} \stackrel{\text { def }}{=} \sum_{x \in \mathbf{Z}} x^{2} a(x)<\infty \quad \text { и } \quad \mathbf{E} \xi=f^{\prime}(1)=1, \quad \sigma^{2} \stackrel{\text { def }}{=} \mathbf{D} \xi^{2} \in(0, \infty) .
$$

Таким образом, рассматриваемый нами процесс ветвления является критическим с конечной дисперсией числа непосредственных потомков одной частицы. Положим

$$
K \stackrel{\text { def }}{=} \frac{2^{3 / 4}}{\sigma \pi^{1 / 4}} \sqrt{\frac{b(1-\alpha)}{\alpha}} \quad \text { и } \quad c_{q} \stackrel{\text { def }}{=} \pi K^{2}=\frac{2 \sqrt{2 \pi} b(1-\alpha)}{\sigma^{2} \alpha} .
$$

Теорема 1 (см. [1]). Если выполнены условия (2), то при $t \rightarrow \infty$

$$
Q(t) \stackrel{\text { def }}{=} \mathbf{P}\{\mu(t)>0\} \sim K t^{-1 / 4}, \quad q(t) \stackrel{\text { def }}{=} \mathbf{P}\{\zeta(t)>0\} \sim \frac{c_{q}}{\sqrt{t} \ln t}
$$

$u$, кроме того, для всех $s \in[0,1]$

$$
\lim _{t \rightarrow \infty} \mathbf{E}\left[s^{\mu(t)} \mid \eta(t)>0\right]=1-\sqrt{1-s} .
$$

Следствие 1 (см. [1]). Если выполнень условия (2), то $\mathbf{P}\{\eta(t)>$ $0\} \sim K t^{-1 / 4}$ при $t \rightarrow \infty u$, кроме того, для всех $s \in[0,1]$

$$
\lim _{t \rightarrow \infty} \mathbf{E}\left[s^{\eta(t)} \mid \eta(t)>0\right]=1-\sqrt{1-s} .
$$

Теорема 2 (см. [2]). Если выполнень условия (2), то при всех $\lambda \in[0, \infty)$

$$
\lim _{t \rightarrow \infty} \mathbf{E}\left\{\exp \left\{-\frac{\lambda \zeta(t)}{\mathbf{E}[\zeta(t) \mid \zeta(t)>0]}\right\} \mid \zeta(t)>0\right\}=\frac{1}{\lambda+1} .
$$

Приведенные утверждения не содержат информации о распределении случайной величины $\mu(t)$ в такие (редкие, как показывает теорема 1 ) моменты времени, когда $\zeta(t)>0$. В данной работе мы восполняем этот пробел и, среди прочего, описываем асимптотическое поведение $\mu(t)$ при условии $\zeta(t)>0$. Для формулировки основного результата статьи - 
теоремы 3 нам понадобится функция $\varphi\left(\lambda_{1}\right)$, являющаяся непрерывным положительным решением уравнения

$$
\varphi\left(\lambda_{1}\right)=1-\alpha \sigma^{2} \lambda_{1}^{1 / 2} \int_{0}^{1} \varphi^{2}\left(\lambda_{1}(1-v)\right) d v^{1 / 2}, \quad \lambda_{1} \geqslant 0
$$

(которое, в соответствии с леммой 7 из [3], существует, единственно и, более того, допускает единственное аналитическое продолжение на область $\left.\operatorname{Re} \lambda_{1}>0\right)$. Аналогично определим функцию $D\left(\lambda_{2}\right)$ как единственное ограниченное рециение уравнения

$$
D\left(\lambda_{2}\right)=1-\alpha \sigma^{2} \lambda_{2} \int_{0}^{1} \frac{D\left(\lambda_{2} \sqrt{y}\right) \varphi\left(\lambda_{2}^{2} y\right)}{\sqrt{y(1-y)}} d y, \quad \lambda_{2} \geqslant 0,
$$

которое будет положительным и имеющим аналитическое продолжение на область $\operatorname{Re} \lambda_{2}>0$. Существование и единственность такого решения будут установлены в лемме 3 и в ходе доказательства формулируемой ниже теоремы 3.

Теорема 3. Если выполнень условия (2) и

$$
h^{\prime \prime}(x)=\sigma^{2}+o\left(\left|\ln ^{-1} x\right|\right) \quad \text { при } \quad x \rightarrow+0,
$$

mо для всех $\lambda_{1}, \lambda_{2} \geqslant 0$

$$
\lim _{t \rightarrow \infty} \mathbf{E}\left\{\exp \left\{-\frac{\lambda_{1} \zeta(t)}{\mathbf{E}[\zeta(t) \mid \zeta(t)>0]}-\frac{\lambda_{2} \mu(t)}{c_{p} \sqrt{t}}\right\} \mid \zeta(t)>0\right\}=\frac{D\left(\lambda_{2}\right)}{1+\lambda_{1}}
$$

zде $c_{p}^{-1} \stackrel{\text { def }}{=} b \sqrt{2 \pi}(1-\alpha)$.

Таким образом, случайные величины $\zeta(t)$ и $\mu(t)$ - количество частиц вне нуля и в нуле в момент $t$, - нормированные соответствующим образом, асимптотически независимы в такие моменты $t$, когда $\zeta(t)>0$.

Прежде чем переходить к доказательству теоремы 3 , опишем асимптотическое поведение нормирующей функции $\mathbf{E}\{\zeta(t) \mid \zeta(t)>0\}$ при $t \rightarrow \infty$. С этой целью рассмотрим порождающее случайное блуждание по решетке $\mathbf{Z}$ без ветвления. Предположим, что блуждание начинается в нуле в момент времени $t=0$, и пусть $\tau_{1}$ - время, проведенное случайным блужданием в нуле до момента первого выхода из него, а $\tau_{2}$ промежуток времени между моментом первого выхода частицы из нуля и моментом ее первого возврашения в нуль. Отметим, что в силу определения $a(0,0)=-1$, и, следовательно, $G_{1}(t) \stackrel{\text { def }}{=} \mathbf{P}\left\{\tau_{1} \leqslant t\right\}=1-e^{-t}$. Положим

$$
G_{2}(t) \stackrel{\text { def }}{=} \mathbf{P}\left\{\tau_{2} \leqslant t\right\}, \quad G_{3}(t) \stackrel{\text { def }}{=} \alpha G_{1}(t)+(1-\alpha) G_{1} * G_{2}(t),
$$

где символ * обозначает операцию свертки распределений. Как показано в [1], функция $P(t) \stackrel{\text { def }}{=} \mathbf{E} \zeta(t)$ допускает представление

$$
P(t)=\left(1-G_{1}(\cdot)\right) * U(t)
$$


где

$$
U(t) \stackrel{\text { def }}{=} \sum_{k=0}^{\infty} G_{3}^{* k}(t)
$$

$G_{3}^{* 0}(t)=1$, a $G_{3}^{* k}(t), k \geqslant 1$, является $k$-кратной сверткой функции $G_{3}(t)$ с самой собой.

Оказывается, что функция $P(t)$ обладает рядом приятных свойств.

Лемма 1 (см. [1]). Функция $P(t)$ монотонно убьвает и имеет асимптотическое представление $P(t) \sim c_{p} t^{-1 / 2}$ nрu $t \rightarrow \infty$.

Последнее утверждение в сочетании с теоремой 1 означает, что

$$
\mathbf{E}[\zeta(t) \mid \zeta(t)>0]=\frac{\mathbf{E} \zeta(t)}{\mathbf{P}\{\zeta(t)>0\}}=\frac{P(t)}{q(t)} \sim c^{*} \ln t, \quad t \rightarrow \infty,
$$

где $c^{*} \stackrel{\text { def }}{=} c_{p} c_{q}^{-1}=\alpha \sigma^{2}(4 \pi)^{-1} b^{-2}(1-\alpha)^{-2}$.

\section{2. Случайные блуждания и процессы Беллмана-Харриса.} Для доказательства теоремы 3 мы воспользуемся подходом, предложенным в работах [1] и [2]. С этой целью мы введем вспомогательный процесс Беллмана-Харриса $\left(Z_{1}(t), Z_{2}(t)\right)$ с двумя типами частиц (см. [10], [11], [12]), где через $Z_{i}(t), i=1,2$, обозначено количество частиц $i$-го типа в процессе в момент времени $t$. Пусть

$$
F_{i}\left(t ; s_{1}, s_{2}\right) \stackrel{\text { def }}{=} \mathbf{E}\left[s_{1}^{Z_{1}(t)} s_{2}^{Z_{2}(t)} \mid Z_{i}(0)=1\right], \quad i=1,2
$$

обозначают производящие функции вектора $\left(Z_{1}(t), Z_{2}(t)\right)$ в момент $t>0$ при условии, что в нулевой момент времени процесс начался с одной частицы типа $i$. Опишем характеристики необходимого нам критического процесса Беллмана-Харриса с двумя типами частиц. Продолжительность жизни частиц первого типа в этом процессе имеет функцию распределения $G_{1}(t)=\mathbf{P}\left\{\tau_{1} \leqslant t\right\}=1-e^{-t}, t \geqslant 0$, а в момент гибели такая частица порождает потомков двух типов с производящей функцией $f_{1}\left(s_{1}, s_{2}\right)=\alpha f\left(s_{1}\right)+(1-\alpha) s_{2}$. Иными словами, с вероятностью $\alpha f_{k}$ она производит ровно $k=0,1,2, \ldots$ частиц первого типа и с вероятностью $1-\alpha$ ровно одну частицу второго типа, где величины $f_{k}$ те же, что и в определении (1). Продолжительность жизни частиц второго типа имеет распределение $G_{2}(t)=\mathbf{P}\left\{\tau_{2} \leqslant t\right\}$ (что соответствует распределению времени, проведенного частицей каталитического ветвящегося случайного блуждания вне нуля, где она не может порождать потомков, до ее первого возвращения в нуль при условии, что она вышла из нуля). В момент гибели частица второго типа порождает ровно одну частицу первого типа и ничего более. Таким образом, для частиц второго типа производящая функция числа непосредственных потомков имеет вид $f_{2}\left(s_{1}, s_{2}\right)=s_{1}$.

Определенный нами процесс Беллмана-Харриса с двумя типами частиц является критическим и неразложимым, так как максимальный по 
модулю корень его матрицы средних

$$
M \stackrel{\text { def }}{=}\left\|\frac{\partial f_{i}}{\partial s_{j}}(1,1)\right\|_{i, j=1,2}=\left(\begin{array}{cc}
\alpha & 1-\alpha \\
1 & 0
\end{array}\right)
$$

равен 1 , а все элементы матрицы $M^{2}$ положительны. Очевидно, что

$$
\left(Z_{1}(t), Z_{2}(t)\right) \stackrel{\text { distr }}{=}(\zeta(t), \mu(t)) .
$$

Используя явный вид функций $f_{i}\left(s_{1}, s_{2}\right), i=1,2$, запишем стандартные интегральные уравнения для производящих функций описанного ветвяшегося процесса (см., например, [10, гл. VIII, §1], или [11]):

$$
\begin{aligned}
& F_{1}\left(t ; s_{1}, s_{2}\right)=s_{1}\left(1-G_{1}(t)\right) \\
& \quad+\int_{0}^{t}\left(\alpha f\left(F_{1}\left(t-u ; s_{1}, s_{2}\right)\right)+(1-\alpha) F_{2}\left(t-u ; s_{1}, s_{2}\right)\right) d G_{1}(u) \\
& F_{2}\left(t ; s_{1}, s_{2}\right)=s_{2}\left(1-G_{2}(t)\right)+\int_{0}^{t} F_{1}\left(t-u ; s_{1}, s_{2}\right) d G_{2}(u) .
\end{aligned}
$$

Подставляя выражение для $F_{2}\left(t ; s_{1}, s_{2}\right)$ из второго уравнения в первое, получаем

$$
\begin{aligned}
F_{1}\left(t ; s_{1}, s_{2}\right)= & s_{1}\left(1-G_{1}(t)\right)+s_{2}(1-\alpha)\left(1-G_{2}(\cdot)\right) * G_{1}(t) \\
& +\int_{0}^{t} \alpha f\left(F_{1}\left(t-u ; s_{1}, s_{2}\right)\right) d G_{1}(u) \\
& +\int_{0}^{t}(1-\alpha) F_{1}\left(t-u ; s_{1}, s_{2}\right) d\left(G_{1} * G_{2}(u)\right) .
\end{aligned}
$$

Полагая $Q\left(t ; s_{1}, s_{2}\right) \stackrel{\text { def }}{=} 1-F_{1}\left(t ; s_{1}, s_{2}\right)$, мы видим, что

$$
\begin{aligned}
Q\left(t ; s_{1}, s_{2}\right)= & \left(1-s_{1}\right)\left(1-G_{1}(t)\right)+\left(1-s_{2}\right)(1-\alpha)\left(1-G_{2}(\cdot)\right) * G_{1}(t) \\
& +\int_{0}^{t} Q\left(t-u ; s_{1}, s_{2}\right) d G_{3}(u) \\
& -\alpha \int_{0}^{t} h\left(Q\left(t-u ; s_{1}, s_{2}\right)\right) d G_{1}(u)
\end{aligned}
$$

где $h(x) \stackrel{\text { def }}{=} f(1-x)-(1-x)$, а функция $G_{3}(t)$ та же, что и в $(6)$.

В силу (7) и (8) решение уравнения восстановления (11) можно записать ь виде

$$
\begin{aligned}
Q\left(t ; s_{1}, s_{2}\right)= & \left(1-s_{1}\right) P(t)+(1-\alpha)\left(1-s_{2}\right)\left(1-G_{2}(\cdot)\right) * G_{1} * U(t) \\
& -\alpha \int_{0}^{t} h\left(Q\left(t-u ; s_{1}, s_{2}\right)\right) d\left(G_{1} * U(u)\right) .
\end{aligned}
$$

Заметим, что $(1-\alpha)\left(1-G_{2}(\cdot)\right) * G_{1}(t)=(1-\alpha) G_{1}(t)-(1-\alpha) G_{2} * G_{1}(t)=$ $G_{1}(t)-G_{3}(t)$ и в силу $(6)$

$$
(1-\alpha)\left(1-G_{2}(\cdot)\right) * G_{1} * U(t)=G_{1} * U(t)-G_{3} * U(t)=1-P(t) .
$$


Учитывая, что $G_{1}(t)=1-e^{-t}$, получаем $\left(G_{1} * U(t)\right)^{\prime}=\left(1-G_{1}(\cdot)\right) *$ $U(t)=P(t)$. Используя полученные выше соотношения, преобразуем уравнение (12) к виду

$$
\begin{aligned}
Q\left(t ; s_{1}, s_{2}\right)= & \left(1-s_{1}\right) P(t)+(1-P(t))\left(1-s_{2}\right) \\
& -\alpha \int_{0}^{t} h\left(Q\left(t-u ; s_{1}, s_{2}\right)\right) P(u) d u .
\end{aligned}
$$

Пусть $Q(t ; s, 1) \stackrel{\text { def }}{=} q(t ; s)$. Тогда $q(t)=\mathbf{P}\{\zeta(t)>0\}=q(t ; 0)$. Подставляя в (13) $s_{1}=s$ и $s_{2}=1$, получаем уравнение

$$
q(t ; s)=(1-s) P(t)-\alpha \int_{0}^{t} h(q(t-u ; s)) P(u) d u .
$$

3. Совместное распределение. Данный пункт посвящен доказательству теоремы 3.

$\mathrm{B}$ дальнейшем для удобства символами $C, C_{1}, C_{2}, \ldots$ будут обозначаться ограниченные положительные постоянные, которые могут быть различными в разных формулах (как правило, в новых формулах постоянные будут нумероваться заново). В частности, соотношение $C_{1} \omega_{1}(t) \leqslant$ $\omega(t) \leqslant C_{2} \omega_{2}(t), t \geqslant t_{0}$, означает, что для функций $\omega(t), \omega_{1}(t)$ и $\omega_{2}(t)$ сушествуют постоянные $C_{1}, C_{2} \in(0, \infty)$ такие, что указанные неравенства верны при всех $t \geqslant t_{0}$.

Лемма 2. Для всех $s_{1}, s_{2} \in[0,1]$ вернь оченки

$$
\begin{aligned}
0 & \leqslant Q\left(t ; s_{1}, s_{2}\right)-q\left(t ; s_{1}\right) \leqslant 1-s_{2} \quad u \\
0 & \leqslant\left(Q\left(t ; s_{1}, s_{2}\right)-q\left(t ; s_{1}\right)\right)-\left(Q\left(t ; 0, s_{2}\right)-q(t ; 0)\right) \\
& =\left(q(t ; 0)-q\left(t ; s_{1}\right)\right)-\left(Q\left(t ; 0, s_{2}\right)-Q\left(t ; s_{1}, s_{2}\right)\right) .
\end{aligned}
$$

Д ок а з а т е л ь с т в о. По определению

$$
Q\left(t ; s_{1}, s_{2}\right)=1-\mathbf{E} s_{1}^{Z_{1}(t)} s_{2}^{Z_{2}(t)} \geqslant 1-\mathbf{E} s_{1}^{Z_{1}(t)}=Q\left(t ; s_{1}, 1\right)=q\left(t ; s_{1}\right) .
$$

Из последнего соотношения и равенства (13) заключаем, что

$$
\begin{aligned}
0 \leqslant & Q\left(t ; s_{1}, s_{2}\right)-Q\left(t ; s_{1}, 1\right)=Q\left(t ; s_{1}, s_{2}\right)-q\left(t ; s_{1}\right)=\left(1-s_{2}\right)(1-P(t)) \\
& +\alpha \int_{0}^{t}\left(h\left(q\left(t-u ; s_{1}\right)\right)-h\left(Q\left(t-u ; s_{1}, s_{2}\right)\right)\right) P(u) d u \\
\leqslant & \left(1-s_{2}\right)(1-P(t)),
\end{aligned}
$$

что доказывает $(14)$.

Для доказательства второй части леммы 2 воспользуемся определением функции $Q\left(t ; s_{1}, s_{2}\right)$, согласно которому

$$
\begin{aligned}
& \left(Q\left(t ; s_{1}, s_{2}\right)-q\left(t ; s_{1}\right)\right)-\left(Q\left(t ; 0, s_{2}\right)-q(t ; 0)\right) \\
& \quad=\mathbf{E}\left[\left(1-s_{2}^{Z_{2}(t)}\right) s_{1}^{Z_{1}(t)}\right]-\mathbf{E}\left[\left(1-s_{2}^{Z_{2}}\right) 0^{Z_{1}(t)}\right] \\
& \quad=\mathbf{E}\left[\left(1-s_{2}^{Z_{2}(t)}\right)\left(s_{1}^{Z_{1}(t)}-0^{Z_{1}(t)}\right)\right] \geqslant 0 .
\end{aligned}
$$

Лемма доказана. 
Положим

$$
\begin{aligned}
s_{1}(t)=s_{1}\left(t ; \lambda_{1}\right) & \stackrel{\text { def }}{=} \exp \left\{-\frac{\lambda_{1} q(t)}{P(t)}\right\}=\exp \left\{-\frac{\lambda_{1}}{\mathbf{E}[\zeta(t) \mid \zeta(t)>0]}\right\}, \\
s_{2}(t)=s_{2}\left(t ; \lambda_{2}\right) & \stackrel{\text { def }}{=} \exp \left\{-\frac{\lambda_{2}}{c_{p} \sqrt{t}}\right\}, \\
\Delta_{1}\left(u, t ; \lambda_{2}\right) & \stackrel{\text { def }}{=} Q\left(u ; 0, s_{2}(t)\right)-q(u ; 0), \\
\Delta_{1}\left(t ; \lambda_{2}\right) & \stackrel{\text { def }}{=} \Delta_{1}\left(t, t ; \lambda_{2}\right), \\
\Delta_{2}\left(u, t ; \lambda_{1}, \lambda_{2}\right) & \stackrel{\text { def }}{=} Q\left(u ; s_{1}(t), s_{2}(t)\right)-q\left(u ; s_{1}(t)\right), \\
\Delta_{2}\left(t ; \lambda_{1}, \lambda_{2}\right) & \stackrel{\text { def }}{=} \Delta_{2}\left(t, t ; \lambda_{1}, \lambda_{2}\right) .
\end{aligned}
$$

Отметим, что в силу (17) $s_{2}\left(t ; \lambda_{2}\right)=s_{2}\left(t \lambda_{2}^{-2} ; 1\right)$. Отсюда, учитывая обозначентя (16) и (20) и определение функции $Q\left(t ; s_{1}, s_{2}\right)$, получаем

$$
\Delta_{2}\left(y t, t ; \lambda_{1}, \lambda_{2}\right)=\Delta_{2}\left(y t, y t ; \dot{\lambda}_{1} \frac{q(t) P(y t)}{P(t) q(y t)}, \lambda_{2} \sqrt{y}\right) .
$$

Введем величины

$$
\begin{aligned}
& \Delta\left(u, t ; \lambda_{1}, \lambda_{2}\right) \stackrel{\text { def }}{=} \Delta_{2}\left(u, t ; \lambda_{1}, \lambda_{2}\right)-\Delta_{1}\left(u, t ; \lambda_{2}\right) \\
&= q(u ; 0)-q\left(u ; s_{1}\left(t ; \lambda_{1}\right)\right) \\
&+\left(Q\left(u ; s_{1}\left(t ; \lambda_{1}\right), s_{2}\left(t ; \lambda_{2}\right)\right)\right. \\
&\left.\quad Q\left(u ; 0, s_{2}\left(t ; \lambda_{2}\right)\right)\right) \geqslant 0, \\
& \Delta\left(t ; \lambda_{1}, \lambda_{2}\right) \stackrel{\text { def }}{=} \Delta_{2}\left(t ; \lambda_{1}, \lambda_{2}\right)-\Delta_{1}\left(t ; \lambda_{2}\right) \geqslant 0,
\end{aligned}
$$

неотрицательность которых следует из оценки (15).

Теперь мы можем наметить схему доказательства теоремы 3.

Несложно проверить, что

$$
\begin{aligned}
H_{t}\left(\lambda_{1}, \lambda_{2}\right) & \stackrel{\text { def }}{=} \mathbf{E}\left[s_{1}^{Z_{1}(t)}(t) s_{2}^{Z_{2}(t)}(t) \mid Z_{1}(t)>0\right] \\
& =\frac{F\left(t ; s_{1}(t), s_{2}(t)\right)-F\left(t ; 0, s_{2}(t)\right)}{P\left(Z_{1}(t)>0\right)} \\
& =\frac{Q\left(t ; 0, s_{2}(t)\right)-Q\left(t ; s_{1}(t), s_{2}(t)\right)}{q(t)}=\Theta_{t}\left(\lambda_{1}\right)-\Psi_{t}\left(\lambda_{1}, \lambda_{2}\right),
\end{aligned}
$$

где

$$
\Theta_{t}\left(\lambda_{1}\right) \stackrel{\text { def }}{=} \frac{q(t)-q\left(t ; s_{1}(t)\right)}{q(t)}, \quad \Psi_{t}\left(\lambda_{1}, \lambda_{2}\right) \stackrel{\text { def }}{=} \frac{\Delta\left(t ; \lambda_{1}, \lambda_{2}\right)}{q(t)} .
$$

Отметим, что ввиду (24) и $(25) 0 \leqslant \Psi_{t}\left(\lambda_{1}, \lambda_{2}\right) \leqslant 1$ при $\lambda_{1} \geqslant 0, \lambda_{2} \geqslant 0$ и, кроме того, справедливо представление

$$
\begin{aligned}
\frac{\Delta\left(t y, t ; \lambda_{1}, \lambda_{2}\right)}{q(t y)} & =[q(t y)]^{-1} \Delta\left(t y ; \lambda_{1} \frac{q(t) P(t y)}{\left.q(t y) P(t), \lambda_{2} \sqrt{y}\right)}\right. \\
& =\Psi_{t y}\left(\lambda_{1} \frac{q(t) P(t y)}{q(t y) P(t)}, \lambda_{2} \sqrt{y}\right) .
\end{aligned}
$$


По теореме 2 в силу совпадения распределений в (10)

$$
\lim _{t \rightarrow \infty} \Theta_{t}\left(\lambda_{1}\right)=\frac{1}{1+\lambda_{1}} .
$$

Для доказательства теоремы 3 достаточно найти $\lim _{t \rightarrow \infty} \Psi_{t}\left(\lambda_{1}, \lambda_{2}\right)$. Поиск этого предела - непростая задача, и в качестве первого шага на этом пути мы докажем в лемме 3 существование и единственность решения уравнения (4) в некоторой правой окрестности $0 \leqslant \lambda_{2} \leqslant b<\infty$ на действительной оси.

Лемма 3. Уравнение (4) не может иметь более одного решения на полупрямой $\lambda_{2}>0$, причем на полуинтервале $\lambda_{2} \in[0,2 \rho)$, где $\rho=$ $\left(2 \alpha \pi \sigma^{2}\right)^{-1}$, оно имеет в точности одно решение.

Д о к а з а т е л ь с т в о. Пусть $C[0,2 \rho)$ обозначает совокупность всех непрерывных функций на $[0,2 \rho)$. Для функций $T(\cdot) \in C[0,2 \rho)$ введем оператор $\mathscr{L}: C[0,2 \rho) \mapsto C[0,2 \rho)$, задаваемый равенством

$$
\mathscr{L} T\left(\lambda_{2}\right) \stackrel{\text { def }}{=} 1-\alpha \sigma^{2} \lambda_{2} \int_{0}^{1} \frac{T\left(\lambda_{2} \sqrt{y}\right) \varphi\left(\lambda_{2}^{2} y\right) d y}{\sqrt{y(1-y)}} .
$$

Для $\lambda_{2} \in[0,2 \rho)$ положим $T^{0}\left(\lambda_{2}\right) \equiv 1$ и $T^{n+1}\left(\lambda_{2}\right)=\mathscr{L} T^{n}\left(\lambda_{2}\right), n=$ $0,1,2, \ldots$ Используя неравенство $0 \leqslant \varphi(\lambda) \leqslant 1$, следующее из определения (3), и равенство

$$
\int_{0}^{1} \frac{d y}{\sqrt{y(1-y)}}=\pi
$$

получаем, что при $\lambda_{2} \in[0,2 \rho)$

$$
1 \geqslant T^{1}\left(\lambda_{2}\right)=1-\alpha \sigma^{2} \lambda_{2} \int_{0}^{1} \frac{\varphi\left(\lambda_{2}^{2} y\right) d y}{\sqrt{y(1-y)}} \geqslant 1-\alpha \pi \sigma^{2} \lambda_{2} \geqslant 0
$$

и, по индукции, что при всех $n \geqslant 1$

$$
1 \geqslant T^{n+1}\left(\lambda_{2}\right)=1-\alpha \sigma^{2} \lambda_{2} \int_{0}^{1} \frac{T^{n}\left(\lambda_{2} \sqrt{y}\right) \varphi\left(\lambda_{2}^{2} y\right) d y}{\sqrt{y(1-y)}} \geqslant 1-\alpha \pi \sigma^{2} \lambda_{2} \geqslant 0 .
$$

С помощью (29) несложно показать по индукции, что для $\lambda_{2} \in[0,2 \rho)$

$$
\begin{aligned}
\left|T^{n+1}\left(\lambda_{2}\right)-T^{n}\left(\lambda_{2}\right)\right| & \leqslant \alpha \sigma^{2} \lambda_{2} \int_{0}^{1}\left|T^{n}\left(\lambda_{2} \sqrt{y}\right)-T^{n-1}\left(\lambda_{2} \sqrt{y}\right)\right| \frac{\varphi\left(\lambda_{2}^{2} y\right) d y}{\sqrt{y(1-y)}} \\
& \leqslant \alpha \sigma^{2} \lambda_{2} \sup _{0 \leqslant z \leqslant \lambda_{2}}\left|T^{n}(z)-T^{n-1}(z)\right| \int_{0}^{1} \frac{\varphi\left(\lambda_{2}^{2} y\right) d y}{\sqrt{y(1-y)}} \\
& \leqslant \frac{\lambda_{2}}{2 \rho} \sup _{0 \leqslant z \leqslant \lambda_{2}}\left|T^{n}(z)-T^{n-1}(z)\right| \leqslant\left(\frac{\lambda_{2}}{2 \rho}\right)^{n+1}
\end{aligned}
$$

Таким образом, последовательность функций $\left\{T^{n}\left(\lambda_{2}\right)\right\}_{n \geqslant 1}$ фундаментальна на множестве $\lambda_{2} \in[0,2 \rho)$. Следовательно, уравнение (4) имеет по крайней мере одно решение на этом множестве. Предположим, что существует два различных решения $T^{*}\left(\lambda_{2}\right)$ и $T^{* *}\left(\lambda_{2}\right)$ уравнения (4) на 
полуинтервале $0 \leqslant \lambda_{2}<2 \rho$. Ясно, что

$$
\begin{aligned}
\left|T^{*}\left(\lambda_{2}\right)-T^{* *}\left(\lambda_{2}\right)\right| & \leqslant \alpha \sigma^{2} \lambda_{2} \int_{0}^{1}\left|T^{*}\left(\lambda_{2} \sqrt{y}\right)-T^{* *}\left(\lambda_{2} \sqrt{y}\right)\right| \frac{\varphi\left(\lambda_{2}^{2} y\right)}{\sqrt{y(1-y)}} d y \\
& \leqslant \alpha \sigma^{2} \lambda_{2} \sup _{0 \leqslant z \leqslant \lambda_{2}}\left|T^{*}(z)-T^{* *}(z)\right| \int_{0}^{1} \frac{\varphi\left(\lambda_{2}^{2} y\right)}{\sqrt{y(1-y)}} d y \\
& \leqslant \frac{\lambda_{2}}{2 \rho} \sup _{0 \leqslant z \leqslant \lambda_{2}}\left|T^{*}(z)-T^{* *}(z)\right|,
\end{aligned}
$$

что в силу принципа сжимающих отображений означает существование на полуинтервале $0 \leqslant \lambda_{2}<2 \rho$ не более одного решения уравнения (4). Полученное противоречие показывает, что уравнение (4) имеет в указанной области ровно одно решение.

Вторая часть леммы 3 доказана, а для доказательства первой нам необходимо следующее утверждение.

Лемма 4. На положительной полуоси $\lambda_{2}>0$ уравнение

$$
\psi\left(\lambda_{2}\right)=\alpha \sigma^{2} \lambda_{2} \int_{0}^{1} \psi\left(\lambda_{2} \sqrt{y}\right) \frac{\varphi\left(\lambda_{2}^{2} y\right)}{\sqrt{y(1-y)}} d y
$$

имеет единственное ограниченное решение: $\psi\left(\lambda_{2}\right) \equiv 0$.

Д о к а з а т е л ь с т в о. Прежде всего отметим, что если обозначить разность $T^{*}\left(\lambda_{2}\right)-T^{* *}\left(\lambda_{2}\right)$ через $\psi\left(\lambda_{2}\right)$, то только что приведенное в лемме 3 доказательство единственности решения уравнения (4) на полуинтервале $\lambda_{2} \in[0,2 \rho)$ означает, что на данном полуинтервале любое решение $\psi\left(\lambda_{2}\right) \equiv 0$. Ясно также, что $\psi\left(\lambda_{2}\right) \equiv 0$ является решением нашего уравнения.

Допустим теперь, что есть ненулевое ограниченное решение $\psi\left(\lambda_{2}\right)$ интересующего нас уравнения. При этом предположении параметр $\rho_{0} \stackrel{\text { def }}{=}$ $\sup \{\lambda: \psi(x)=0, \forall x<\lambda\}$ конечен и уравнение (30) принимает вид

$$
\psi\left(\lambda_{2}\right)=\alpha \sigma^{2} \lambda_{2} \int_{\rho_{0} / \lambda_{2}}^{1} \psi\left(\lambda_{2} \sqrt{y}\right) \frac{\varphi\left(\lambda_{2}^{2} y\right)}{\sqrt{y(1-y)}} d y .
$$

По аналогии с доказательством единственности из предшествующей леммы, учитывая неравенства $0 \leqslant \varphi(x) \leqslant 1$ при $x \geqslant 0$ и $\sqrt{y(1-y)} \geqslant$ $\sqrt{a} \sqrt{1-y}$ при $y \in[a, 1]$, получаем

$$
\begin{aligned}
\sup _{\rho_{0} \leqslant z \leqslant \lambda_{2}}|\psi(z)| & \leqslant \alpha \sigma^{2} \lambda_{2} \sup _{\rho_{0} \leqslant z \leqslant \lambda_{2}}|\psi(z)| \sqrt{\frac{\lambda_{2}}{\rho_{0}}} \int_{\rho_{0} / \lambda_{2}}^{1} \frac{1}{\sqrt{1-y}} d y \\
& =2 \alpha \sigma^{2} \lambda_{2} \sqrt{\frac{\lambda_{2}-\rho_{0}}{\rho_{0}}} \sup _{\rho_{0} \leqslant z \leqslant \lambda_{2}}|\psi(z)| .
\end{aligned}
$$

Очевидно, что существуют $\lambda_{2}>\rho_{0}$, достаточно близкие к $\rho_{0}$, такие, что $2 \alpha \sigma^{2} \lambda_{2} \sqrt{\left(\lambda_{2}-\rho_{0}\right) / \rho_{0}}<1$. Это в силу предшествующих неравенств влечет $\sup _{\rho_{0} \leqslant z \leqslant \lambda_{2}} \psi(z)=0$, что противоречит определению параметра $\rho_{0}$, если допустить, что он конечен.

Доказательство леммы 4 завершено. 
Лемма 4 позволяет нам завершить доказательство леммы 3 . В самом деле, если допустить существование двух различных решений уравнения (4) $D_{1}\left(\lambda_{2}\right) \neq D_{2}\left(\lambda_{2}\right)$, то их разность $\psi\left(\lambda_{2}\right)=D_{1}\left(\lambda_{2}\right)-D_{2}\left(\lambda_{2}\right)$ является решением уравнения из леммы 4, которое, как мы знаем, обязано быть тождественным нулем.

Лемма 3 доказана.

Положим

$$
I\left(t ; s_{1}, s_{2}\right) \stackrel{\text { def }}{=} \alpha \int_{0}^{t}\left(h\left(Q\left(t-u ; s_{1}, s_{2}\right)\right)-h\left(q\left(t-u ; s_{1}\right)\right)\right) P(u) d u .
$$

Из представления (13) и определений (18)-(21) и (23) следует, что

$$
\begin{aligned}
\Delta_{1}\left(t ; \lambda_{2}\right) & =(1-P(t))\left(1-s_{2}(t)\right)-I\left(t ; 0, s_{2}(t)\right), \\
\Delta_{2}\left(t ; \lambda_{1}, \lambda_{2}\right) & =(1-P(t))\left(1-s_{2}(t)\right)-I\left(t ; s_{1}(t), s_{2}(t)\right)
\end{aligned}
$$

и

$$
\Delta\left(t ; \lambda_{1}, \lambda_{2}\right)=I\left(t ; 0, s_{2}(t)\right)-I\left(t ; s_{1}(t), s_{2}(t)\right) .
$$

Таким образом, изучение асимптотического поведения $\Delta\left(t ; \lambda_{1}, \lambda_{2}\right)$ сводится к исследованию свойств $I\left(t ; s_{1}, s_{2}\right)$ при подходящем выборе $s_{1}$ и $s_{2}$. Положим $y=Q\left(t-u ; s_{1}, s_{2}\right)$ и $x=q\left(t-u ; s_{1}\right)=Q\left(t-u ; s_{1}, 1\right)$, а затем применим формулу Тейлора

$$
h(y)-h(x)=h^{\prime}(x)(y-x)+h^{\prime \prime}(\theta) \frac{(y-x)^{2}}{2},
$$

где $\theta=\theta(x, y) \in[x, y]$, а $h^{\prime \prime}(x) \sim \sigma^{2}$ при $x \rightarrow+0$ в силу условия (5). В результате мы получаем представление

$$
I\left(t ; s_{1}, s_{2}\right)=I_{1}\left(t ; s_{1}, s_{2}\right)+I_{2}\left(t ; s_{1}, s_{2}\right),
$$

где

$$
\begin{aligned}
& I_{1}\left(t ; s_{1}, s_{2}\right) \\
& \stackrel{\text { def }}{=} \alpha \int_{0}^{t} h^{\prime}\left(q\left(t-u ; s_{1}\right)\right)\left(Q\left(t-u ; s_{1}, s_{2}\right)-q\left(t-u ; s_{1}\right)\right) P(u) d u \\
& \begin{aligned}
I_{2}\left(t ; s_{1}, s_{2}\right) \stackrel{\text { def }}{=} \frac{\alpha}{2} \int_{0}^{t} h^{\prime \prime}\left(\theta\left(t-u ; s_{1}, s_{2}\right)\right) \\
\quad \times\left(Q\left(t-u ; s_{1}, s_{2}\right)-q\left(t-u ; s_{1}\right)\right)^{2} P(u) d u
\end{aligned}
\end{aligned}
$$

причем

$$
\theta\left(v ; s_{1}, s_{2}\right) \in\left[q\left(v ; s_{1}\right), Q\left(v ; s_{1}, s_{2}\right)\right] .
$$

Наша следуюшая цель - изучить свойства $I_{i}\left(t ; s_{1}, s_{2}\right), i=1,2$. В дальнейшем символы $\mathscr{K}, \mathscr{K}_{1}$ будут использоваться для обозначения любых фиксированных отрезков $[0, b], 0<b<\infty$, символы $\mathscr{K}_{\varepsilon}, \mathscr{K}_{\varepsilon, 1}-$ для обозначения отделенных от нуля фиксированных отрезков $[\varepsilon, b]$, $0<\varepsilon<b<\infty$, а символ $\mathscr{K}^{2}$ - для обозначения ограниченных фиксированных прямоугольников $\left[0, b_{1}\right] \times\left[0, b_{2}\right]$ из первого квадранта плоскости. 
Лемма 5. Если выполнены условия (2), то для любого $\varepsilon \in(0,1]$

$$
R \stackrel{\text { def }}{=}\left[1+\left(1-s_{1}(t ; \lambda)\right) \frac{P(v)}{q(v)}\right] \frac{q\left(v ; s_{1}(t ; \lambda)\right)}{P(v)\left(1-s_{1}(t ; \lambda)\right)} \longrightarrow 1, \quad t \rightarrow \infty,
$$

равномерно по $t \geqslant v \geqslant t^{\varepsilon} u \lambda \in \mathscr{K}_{\varepsilon}$.

3 а м е ч а н и е 1. Асимптотическое представление (9) и определение (16) позволяют переписать утверждение леммы 5 в виде

$$
\begin{aligned}
q\left(v ; s_{1}(t ; \lambda)\right) & \sim \frac{P(v)\left(1-s_{1}(t ; \lambda)\right)}{1+\left(1-s_{1}(t ; \lambda)\right)(P(v) / q(v))} \\
& \sim \frac{P(v) \lambda}{c^{*} \ln t(1+\lambda(\ln v) / \ln t)}
\end{aligned}
$$

при $t \rightarrow \infty$ равномерно по $t \geqslant v \geqslant t^{\varepsilon}$ и $\lambda \in \mathscr{K}_{\varepsilon}$.

Д ок а з а т ел ь с т в л е м мы 5. В соответствии с (27), при всех $\lambda \geqslant 0$

$$
\lim _{t \rightarrow \infty} \frac{q\left(t ; s_{1}(t ; \lambda)\right)}{q(t)}=\frac{\lambda}{\lambda+1} .
$$

Так как выражение, стоящее под знаком предела в (37), монотонно по $\lambda$ и предельная функция ограничена, монотонна, равномерно непрерывна по $\lambda \in[0, \infty)$ и стремится к 1 при $\lambda \rightarrow \infty$, то сходимость в (37) равномерна по $\lambda \in[0, \infty)$. Поэтому для любого $\varepsilon>0$

$$
\sup _{\lambda \in \mathscr{K}_{\varepsilon}}\left|\frac{(\lambda+1) q\left(t ; s_{1}(t ; \lambda)\right)}{\lambda q(t)}-1\right| \longrightarrow 0, \quad t \rightarrow \infty .
$$

Заметим, что

$$
s_{1}(t ; \lambda)=\exp \left\{-\lambda \frac{q(v)}{P(v)} \frac{q(t)}{P(t)} \frac{P(v)}{q(v)}\right\}=s_{1}\left(v ; \lambda \frac{q(t)}{P(t)} \frac{P(v)}{q(v)}\right)
$$

и при $t \geqslant v \geqslant t^{\varepsilon} \rightarrow \infty$

$$
\lambda \frac{q(t)}{P(t)} \frac{P(v)}{q(v)} \sim \lambda \frac{\ln v}{\ln t} \geqslant C \lambda \frac{\ln v}{\ln t} \geqslant C \lambda \varepsilon .
$$

Следовательно, при $t>2$ параметр $\lambda_{1}=\lambda \frac{q(t)}{P(t)} \frac{P(v)}{q(v)}$ содержится в некотором отрезке $\mathscr{K}_{\varepsilon_{1}, 1}$, если $\lambda \in \mathscr{K}_{\varepsilon}$. Этот факт и равномерность оценки (38) влекут

$$
\sup _{\lambda \in \mathscr{K}_{\epsilon}}\left|\frac{\left[\frac{q(t)}{P(t)} \frac{P(v)}{q(v)} \lambda+1\right] q\left(v ; s_{1}\left(v ; \lambda \frac{q(t)}{P(t)} \frac{P(v)}{q(v)}\right)\right)}{\lambda \frac{q(t)}{P(t)} \frac{P(v)}{q(v)} q(v)}-1\right| \rightarrow 0, \quad t \rightarrow \infty .
$$

Определение (16) и асимптотическое представление (9) дают 1 $s_{1}(t, \lambda) \sim \lambda q(t) / P(t), t \rightarrow \infty$, равномерно по $\lambda \in \mathscr{K}_{\varepsilon}$. Из двух последних асимптотических соотношений вытекает, что

$$
R \sim \frac{\left[1+\lambda \frac{q(t)}{P(t)} \frac{P(v)}{q(v)}\right] q\left(v ; s_{1}(t ; \lambda)\right)}{\lambda q(v)} \frac{P(t)}{q(t)} \frac{q(v)}{P(v)} \longrightarrow 1, \quad t \rightarrow \infty,
$$


равномерно по $\lambda \in \mathscr{K}_{\varepsilon}$. Отсюда, учитывая (9), заключаем, что

$$
q(v ; s(t ; \lambda)) \sim \frac{\left(1-s_{1}(t ; \lambda)\right) P(v)}{1+\left(1-s_{1}(t ; \lambda)\right)(P(v) / q(v))} \sim \frac{P(v)}{c^{*} \ln t[1+\lambda(\ln v / \ln t)]}
$$

при $t \geqslant v \geqslant t^{\varepsilon} \rightarrow \infty$ равномерно по $\lambda \in \mathscr{K}_{\varepsilon}$.

Доказательство леммы завершено.

Отметим, что, согласно теореме 1 , соотношение (36) верно и при $s_{1}(t ; \infty)=0$.

В доказываемой ниже лемме условимся считать, что $\Delta_{2}\left(t ; \lambda_{1}, 0\right) /(1-$ $\left.s_{2}(t ; 0)\right)=\Delta_{1}(t ; 0) /\left(1-s_{2}(t ; 0)\right)=1$.

Лемма 6. Пусть $\varphi(\lambda)$ - решение уравнения (3). Если вылолнень условия (2), то

$$
\frac{\Delta_{2}\left(t ; \lambda_{1}, \lambda_{2}\right)}{1-s_{2}(t ; 1)}-\lambda_{2} \varphi\left(\lambda_{2}^{2}\right) \longrightarrow 0, \quad t \rightarrow \infty,
$$

равномерно относительно $\left(\lambda_{1}, \lambda_{2}\right) \in \mathscr{K}^{2} u$

$$
\frac{\Delta_{1}\left(t ; \lambda_{2}\right)}{1-s_{2}(t ; 1)}-\lambda_{2} \varphi\left(\lambda_{2}^{2}\right) \longrightarrow 0, \quad t \rightarrow \infty
$$

равномерно относительно $\lambda_{2} \in \mathscr{K}$.

Д ок а $з$ а т ел ь с т в о. Заметим, что в силу теоремы 1

$$
q\left(t ; s_{1}\right) \leqslant q(t ; 0)=q(t) \leqslant C(t+1)^{-1 / 2} \ln ^{-1}(t+2)
$$

для некоторой постоянной $C>c_{q}$. Далее, для всех $0 \leqslant s_{1,0} \leqslant s_{1,1} \leqslant 1$

$$
\begin{aligned}
Q\left(t ; s_{1,0}, s_{2}\right)-Q\left(t ; s_{1,1}, s_{2}\right) & =\mathbf{E}\left[\left(s_{1,1}^{Z_{1}(t)}-s_{1,0}^{Z_{1}(t)}\right) s_{2}^{Z_{2}(t)}\right] \\
& \leqslant \mathbf{E}\left[s_{1,1}^{Z_{1}(t)}-s_{1,0}^{Z_{1}(t)}\right] \leqslant q(t ; 0) .
\end{aligned}
$$

Из (17) и формулы Тейлора следует, что для любого $\varepsilon>0$

$$
1-s_{2}\left(t ; \lambda_{2}\right) \sim \lambda_{2}\left(1-s_{2}(t ; 1)\right) \sim \lambda_{2} c_{p}^{-1} t^{-1 / 2}, \quad t \rightarrow \infty,
$$

равномерно по $\lambda_{2} \in \mathscr{K}_{\varepsilon}$.

Полагая $\widehat{Q}(t ; s) \stackrel{\text { def }}{=} Q(t ; s, s)$, несложно проверить, опираясь на оценки (39), определения (18)-(21) и теорему 1 , что

$$
\begin{aligned}
\Delta_{2}\left(t ; \lambda_{1}, \lambda_{2}\right) & =\widehat{Q}\left(t ; s_{2}\left(t ; \lambda_{2}\right)\right)+O\left((t+1)^{-1 / 2} \ln ^{-1}(t+2)\right), \\
\Delta_{1}\left(t ; \lambda_{2}\right) & =\widehat{Q}\left(t ; s_{2}\left(t ; \lambda_{2}\right)\right)+O\left((t+1)^{-1 / 2} \ln ^{-1}(t+2)\right) .
\end{aligned}
$$

Отсюда и из асимптотического соотношения (40) вытекает, что для завершения доказательства леммы достаточно показать, что

$$
c_{p} \sqrt{t} \widehat{Q}\left(t ; s_{2}\left(t ; \lambda_{2}\right)\right)-\lambda_{2} \varphi\left(\lambda_{2}^{2}\right) \longrightarrow 0, \quad t \rightarrow \infty
$$


равномерно по $\lambda_{2} \in \mathscr{K}$. Ввиду (13)

$$
\widehat{Q}(t ; s)=1-s-\alpha \int_{0}^{t} h(\widehat{Q}(t-u ; s)) P(u) d u .
$$

Следовательно,

$$
\frac{\widehat{Q}\left(t ; s_{2}\left(t ; \lambda_{2}\right)\right)}{1-s_{2}(t ; 1)} \leqslant \frac{1-s_{2}\left(t ; \lambda_{2}\right)}{1-s_{2}(t ; 1)} \leqslant C \lambda_{2}, \quad t \geqslant 1 .
$$

$\mathrm{C}$ другой стороны, функция $\varphi\left(\lambda_{2}\right)$ ограничена и непрерывна на полуоси $[0, \infty)$ и, в частности, $\lambda_{2} \varphi\left(\lambda_{2}^{2}\right) \rightarrow 0$ при $\lambda_{2} \rightarrow 0$. Объединяя это утверждение с (43), мы видим, что левая часть в (41) пренебрежимо мала при малых $\lambda_{2}$, и нам осталось показать, что для любого $\varepsilon>0$

$$
\frac{\widehat{Q}\left(t ; s_{2}\left(t ; \lambda_{2}\right)\right)}{1-s_{2}(t ; 1)}-\lambda_{2} \varphi\left(\lambda_{2}^{2}\right) \longrightarrow 0, \quad t \rightarrow \infty,
$$

равномерно по $\lambda_{2} \in \mathscr{K}_{\varepsilon}$. В силу (41) и (40) достаточно установить, что

$$
\frac{\widehat{Q}\left(t ; s_{2}\left(t ; \lambda_{2}\right)\right)}{1-s_{2}\left(t ; \lambda_{2}\right)}-\varphi\left(\lambda_{2}^{2}\right) \longrightarrow 0, \quad t \rightarrow \infty,
$$

равномерно по $\lambda_{2} \in \mathscr{K}_{\varepsilon}$. Чтобы проверить этот факт, положим

$$
\varphi(t ; \theta) \stackrel{\text { def }}{=} \frac{\widehat{Q}\left(t ; s_{2}(t ; \sqrt{\theta})\right)}{1-s_{2}(t ; \sqrt{\theta})}=\frac{\widehat{Q}\left(t ; s_{2}\left(t \theta^{-1} ; 1\right)\right)}{1-s_{2}\left(t \theta^{-1} ; 1\right)}, \quad \theta>0 .
$$

Заметим, что

$$
\frac{\widehat{Q}\left(t(1-y) ; s_{2}\left(t \theta^{-1} ; 1\right)\right)}{1-s_{2}\left(t \theta^{-1} ; 1\right)}=\varphi(t(1-y) ; \theta(1-y))
$$

и, в силу (5) и критичности нашего процесса, верно представление

$$
h(x)=\frac{\sigma^{2}}{2} x^{2}+o\left(\frac{x^{2}}{|\ln x|}\right), \quad x \rightarrow+0 .
$$

Отсюда, отталкиваясь от равенства (42) и используя аргументы, применявшиеся при доказательстве леммы 9 из [3], можно вывести, что $\varphi(t ; \theta)=1+\gamma(t ; \theta)-\frac{\alpha \sigma^{2} t}{2}\left[1-s_{2}\left(\frac{t}{\theta} ; 1\right)\right] \int_{0}^{1} \varphi^{2}(t(1-y) ; \theta(1-y)) P(t y) d y$, где $\gamma(t ; \theta) \rightarrow 0$ при $t \rightarrow \infty$ равномерно по значениям $\theta$, принадлежащим произвольному отрезку $\left[\Theta_{0}, \Theta_{1}\right] \subset(0, \infty)$.

Вспоминая асимптотическое соотношение $(40)$ и лемму 1 , получаем

$$
\varphi(t ; \theta)=1+\gamma_{1}(t, \theta)-\alpha \sigma^{2} \theta^{1 / 2} \int_{0}^{1} \varphi^{2}(t(1-y) ; \theta(1-y)) d y^{1 / 2},
$$

где $\gamma_{1}(t, \theta) \rightarrow 0$ при $t \rightarrow \infty$ равномерно по $\theta \in\left[\Theta_{0}, \Theta_{1}\right]$. 
Уравнение (44) совпадает (с точностью до обозначений) с уравнением (46) из [3], и, следовательно, так же как и в [3],

$$
\varphi(t ; \theta) \rightarrow \varphi(\theta), \quad t \rightarrow \infty
$$

для любого $\theta>0$ и, кроме того, функция $\varphi(\theta)$ имеет единственное аналитическое продолжение на область $\operatorname{Re} \theta>0$.

Осталось показать, что доказанная сходимость равномерна по $\theta \in \mathscr{K}$. Однако это очевидно, так как отношение $\widehat{Q}(t ; s) /(1-s)$ монотонно возрастает при $s \uparrow 1$ и не превосходит единицы и, следовательно, отношение $\varphi(t ; \theta)=Q\left(t ; 1, s_{2}(t ; \sqrt{\theta})\right) /\left(1-s_{2}(t ; \sqrt{\theta})\right)$ монотонно убывает при $\theta \rightarrow \infty$ и, кроме того, ограничено на любом отрезке $\mathscr{K}$, поскольку предельная функция $\varphi(\theta)$ равномерно непрерывна на любом конечном интервале.

Для завершения доказательства леммы 6 достаточно положить $\theta=\lambda_{2}^{2}$.

Обозначим через $\mathscr{U}_{t}$ множество всех ограниченных функций $\gamma\left(t ; \lambda_{1}, \lambda_{2}\right), \gamma_{1}\left(t ; \lambda_{1}, \lambda_{2}\right), \ldots$ (с нижним индексом или без него), стремящихся к нулю при $t \rightarrow \infty$ равномерно по $\left(\lambda_{1}, \lambda_{2}\right) \in \mathscr{K}^{2}$. Функции с совпадающими индексами могут быть различны в различных формулах. Для удобства мы также используем запись $\gamma(t) \in \mathscr{U}_{t}$ для функций $\gamma(t)$, которые не зависят от $\left(\lambda_{1}, \lambda_{2}\right)$ и стремятся к нулю на бесконечности.

3 а м е ч а н и е 2 . Пусть $\Delta_{i}^{*}\left(u, t ; \lambda_{1}, \lambda_{2}\right)$ совпадает с $\Delta_{1}\left(u, t ; \lambda_{2}\right)$ при $i=1$ и совпадает с $\Delta_{2}\left(u, t ; \lambda_{1}, \lambda_{2}\right)$ при $i=2$. В силу (39), (40) и (22) утверждение леммы 6 в новых обозначениях можно записать в виде

$$
\begin{aligned}
& \left(1-s_{2}(t ; 1)\right)^{-1} \Delta_{i}^{*}\left(y t, t ; \lambda_{1}, \lambda_{2}\right) \\
& \quad=\frac{1-s_{2}(t y ; 1)}{1-s_{2}(t ; 1)}\left(1-s_{2}(t y ; 1)\right)^{-1} \Delta_{i}^{*}\left(y t, y t ; \lambda_{1} \frac{q(t) P(y t)}{P(t) q(y t)}, \lambda_{2} \sqrt{y}\right) \\
& \quad=\lambda_{2} \varphi\left(\lambda_{2}^{2} y\right)\left(1+\gamma_{1}\left(y t ; \lambda_{1}, \lambda_{2}\right)\right)+\gamma_{2}\left(y t ; \lambda_{1}, \lambda_{2}\right) \\
& \quad=\lambda_{2} \varphi\left(\lambda_{2}^{2} y\right)+\gamma_{3}\left(y t ; \lambda_{1}, \lambda_{2}\right),
\end{aligned}
$$

где $\gamma_{i}\left(t ; \lambda_{1}, \lambda_{2}\right) \in \mathscr{U}_{t}, i=1,2,3$, причем последнее равенство верно, так как $0 \leqslant \lambda_{2} \varphi\left(\lambda_{2}^{2} y\right) \leqslant \Lambda$, если $\lambda_{2} \in[0, \Lambda]$.

Приступим к изучению асимптотического поведения интегралов (33) и (34) при $t \rightarrow \infty$.

Лемма 7. В условиях теоремь 3 при $t \rightarrow \infty$

$$
\begin{aligned}
\frac{I_{1}\left(t ; 0, s_{2}(t)\right)}{q(t)} & \longrightarrow \alpha \sigma^{2} \lambda_{2} \int_{0}^{1} \frac{\varphi\left(\lambda_{2}^{2} y\right)}{\sqrt{y(1-y)}} d y \\
\frac{I_{1}\left(t ; s_{1}(t), s_{2}(t)\right)}{q(t)} & \longrightarrow \alpha \sigma^{2} \frac{\lambda_{1} \lambda_{2}}{1+\lambda_{1}} \int_{0}^{1} \frac{\varphi\left(\lambda_{2}^{2} y\right)}{\sqrt{y(1-y)}} d y
\end{aligned}
$$

равномерно по $\left(\lambda_{1}, \lambda_{2}\right) \in \mathscr{K}^{2}$. 
Д о к а з а т е л ь с т в о. Из определений (33) и (18) следует, что $I_{1}\left(t ; 0, s_{2}(t)\right)=\alpha \int_{0}^{t} h^{\prime}(q(t-u ; 0)) \Delta_{1}\left(t-u, t ; \lambda_{2}\right) P(u) d u$. Согласно формуле Тейлора, $h^{\prime}(x) \leqslant \sigma^{2} x$ и $h^{\prime}(x) \sim \sigma^{2} x$ при $x \rightarrow+0$. Последние оценки, теорема 1 и леммы 1 и 2 позволяют записать при любом фиксированном $\varepsilon \in\left(0, \frac{1}{2}\right)$ неравенства

$$
\begin{aligned}
& \int_{t(1-\varepsilon)}^{t} h^{\prime}(q(t-u ; 0)) \Delta_{1}\left(t-u, t ; \lambda_{2}\right) P(u) d u \\
& \left.\quad \leqslant \frac{C_{1}}{\sqrt{t+1}}\left(1-s_{2}(t)\right) \int_{t(1-\varepsilon)}^{t} q(t-u ; 0)\right) d u \leqslant \frac{C_{2}}{t+1} \int_{0}^{\varepsilon t} q(u ; 0) d u \\
& \quad \leqslant \frac{C_{3} \sqrt{\varepsilon t}}{(t+1) \ln (\varepsilon t+2)} \leqslant \frac{C \sqrt{\varepsilon}}{\sqrt{t+1} \ln (t+2)} \\
& \int_{0}^{t \varepsilon} h^{\prime}(q(t-u ; 0)) \Delta_{1}\left(t-u, t ; \lambda_{2}\right) P(u) d u \\
& \quad \leqslant \frac{C_{1}}{\sqrt{t+1} \ln (t+2)}\left(1-s_{2}(t)\right) \int_{0}^{t \varepsilon} P(u) d u \\
& \quad \leqslant \frac{C_{2}}{(t+1) \ln (t+2)} \sqrt{\varepsilon t} \leqslant \frac{C \sqrt{\varepsilon}}{\sqrt{t+1} \ln (t+2)}
\end{aligned}
$$

Следовательно, при любом фиксированном $\varepsilon \in\left(0, \frac{1}{2}\right)$

$$
I_{1}\left(t ; 0, s_{2}(t)\right)=I_{\varepsilon, 1}(t)+\sqrt{\varepsilon} O\left(\frac{1}{\sqrt{t+1} \ln (t+2)}\right),
$$

где

$$
\begin{aligned}
I_{\varepsilon, 1}(t) & \stackrel{\text { def }}{=} \alpha \int_{t \varepsilon}^{t(1-\varepsilon)} h^{\prime}(q(t-u ; 0)) \Delta_{1}\left(t-u, t ; \lambda_{2}\right) P(u) d u \\
& =\alpha \sigma^{2} \int_{t \varepsilon}^{t(1-\varepsilon)}(1+\gamma(u)) q(u ; 0) \Delta_{1}\left(u, t ; \lambda_{2}\right) P(t-u) d u \\
& =\alpha \sigma^{2} t \int_{\varepsilon}^{1-\varepsilon}(1+\gamma(y t)) q(y t ; 0) \Delta_{1}\left(y t, t ; \lambda_{2}\right) P((1-y) t) d y,
\end{aligned}
$$

a $\gamma(t) \in \mathscr{U}_{t}$. В силу теоремы 1 и леммы 1 , при $t \rightarrow \infty q(y t ; 0) / q(t ; 0) \rightarrow$ $1 / \sqrt{y}$ и $P((1-y) t) / P(t) \rightarrow 1 / \sqrt{1-y}$ равномерно по $y \in[\varepsilon, 1-\varepsilon]$. Отсюда, учитывая еще раз теорему 1 , лемму 1 , представление $(22)$ с $\lambda_{1}=0$, сходимость интеграла в $(28)$ и то, что отношение $u / t$ отделено от нуля и не превосходит единицы, а также вспоминая соотношение (45), получаем

$$
\begin{aligned}
I_{\varepsilon, 1}(t)= & \left(1+\gamma_{1}\left(t ; \lambda_{2}\right)\right) \alpha \sigma^{2} t\left(1-s_{2}(t ; 1)\right) q(t) P(t) \int_{\varepsilon}^{1-\varepsilon} \frac{\lambda_{2} \varphi\left(\lambda_{2}^{2} y\right)}{\sqrt{y(1-y)}} d y \\
& +\gamma_{2}\left(t ; \lambda_{2}\right) \alpha \sigma^{2}\left(1-s_{2}(t ; 1)\right) t q(t) P(t) \int_{\varepsilon}^{1-\varepsilon} \frac{1}{\sqrt{y(1-y)}} d y \\
= & \left(1+\gamma_{3}\left(t ; \lambda_{2}\right)\right) \alpha \sigma^{2} \lambda_{2} q(t) \int_{\varepsilon}^{1-\varepsilon} \frac{\varphi\left(\lambda_{2}^{2} y\right)}{\sqrt{y(1-y)}} d y+\gamma_{4}\left(t ; \lambda_{2}\right) q(t),
\end{aligned}
$$


где $\gamma_{j}(t ; \lambda) \in \mathscr{U}_{t}, j=1,2,3,4$. Следовательно,

$$
\frac{I_{\varepsilon, 1}(t)}{q(t)} \longrightarrow \alpha \sigma^{2} \lambda_{2} \int_{\varepsilon}^{1-\varepsilon} \frac{\varphi\left(\lambda_{2}^{2} y\right)}{\sqrt{y(1-y)}} d y, \quad t \rightarrow \infty,
$$

равномерно по $\lambda_{2} \in \mathscr{K}$. Последнее совместно с (50) доказывает (46).

Доказательство соотношения (47) проводится по такой же схеме. Мы отметим лишь основные изменения, которые необходимо внести по сравнению с доказательством соотношения (46).

Ввиду оценки $q\left(u ; s_{1}\right) \leqslant q(u ; 0)$ неравенства $(48)$ и (49) остаются справедливыми при замене $q\left(u ; s_{1}(t)\right)$ на $q(u ; 0)$. Далее, по лемме 5 для любых фиксированных $\varepsilon \in\left(0, \frac{1}{2}\right)$ и $y \in(\varepsilon, 1-\varepsilon)$

$$
q\left(y t, \exp \left\{-\frac{\lambda_{1} q(t)}{P(t)}\right\}\right)=q(y t) \frac{\lambda_{1}}{1+\lambda_{1}}(1+\gamma(t ; y))
$$

где $\gamma(t ; y) \rightarrow 0$ при $t \rightarrow \infty$ равномерно по $y \in(\varepsilon, 1-\varepsilon)$.

Отсюда, повторяя аргументы, использованные при изучении свойств интеграла $I_{\varepsilon, 1}(t)$, и применяя (51) вместо теоремы 1 , мы последовательно получаем

$$
\begin{aligned}
I_{\varepsilon, 2}(t) \stackrel{\text { def }}{=} & \alpha t \int_{\varepsilon}^{(1-\varepsilon)} h^{\prime}\left(q\left((1-y) t ; s_{1}\left(t ; \lambda_{1}\right)\right)\right) \Delta_{2}\left((1-y) t, t ; \lambda_{1}, \lambda_{2}\right) P(y t) d y \\
= & \left(1+\gamma_{1}\left(t ; \lambda_{1}, \lambda_{2}\right)\right) \frac{1-s_{2}(t ; 1)}{1+\lambda_{2}} \alpha \sigma^{2} t \lambda_{1} q(t) P(t) \int_{\varepsilon}^{1-\varepsilon} \frac{\lambda_{2} \varphi\left(\lambda_{2}^{2} y\right)}{\sqrt{y(1-y)}} d y \\
& +\gamma_{2}\left(t ; \lambda_{1}, \lambda_{2}\right) \alpha \sigma^{2}\left(1-s_{2}(t ; 1)\right) t q(t) P(t) \int_{\varepsilon}^{1-\varepsilon} \frac{1}{\sqrt{y(1-y)}} d y \\
= & \alpha \sigma^{2} \frac{\lambda_{1} \lambda_{2}}{1+\lambda_{2}}\left(1+\gamma_{3}\left(t ; \lambda_{1}, \lambda_{2}\right)\right) q(t) \int_{\varepsilon}^{1-\varepsilon} \frac{\varphi\left(\lambda_{2}^{2} y\right)}{\sqrt{y(1-y)}} d y \\
& +\gamma_{4}\left(t ; \lambda_{1}, \lambda_{2}\right) q(t)
\end{aligned}
$$

где $\gamma_{j}\left(t ; \lambda_{1}, \lambda_{2}\right) \in \mathscr{U}_{t}, j=1,2,3,4$, и

$$
\frac{I_{\varepsilon, 2}(t)}{q(t)} \longrightarrow \alpha \sigma^{2} \frac{\lambda_{1} \lambda_{2}}{1+\lambda_{1}} \int_{\varepsilon}^{1-\varepsilon} \frac{\varphi\left(\lambda_{2}^{2} y\right)}{\sqrt{y(1-y)}} d y, \quad t \rightarrow \infty,
$$

равномерно по $\left(\lambda_{1}, \lambda_{2}\right) \in \mathscr{K}^{2}$. Последнее в сочетании с определением $I_{1}\left(t ; s_{1}(t), s_{2}(t)\right)$ убеждает нас в справедливости соотношения $(47)$.

Лемма доказана.

Лемма 8. В условиях теоремь 3 для любого Фиксированного $\varepsilon \in\left(0, \frac{1}{2}\right)$

$$
\Psi_{t y}\left(\lambda_{1} \frac{q(t) P(t y)}{q(t y) P(t)}, \lambda_{2} \sqrt{y}\right)-\Psi_{t y}\left(\lambda_{1}, \lambda_{2} \sqrt{y}\right) \longrightarrow 0, \quad t \rightarrow \infty
$$

равномерно по $y \in(\varepsilon, 1-\varepsilon) u\left(\lambda_{1}, \lambda_{2}\right) \in \mathscr{K}^{2}$. 
Доказа т ель с т в о. Используя определение $\Delta\left(u, t ; \lambda_{1}, \lambda_{2}\right)$, данное в (23), и представление (26), имеем

$$
\begin{aligned}
& \Psi_{t y}\left(\lambda_{1} \frac{q(t) P(t y)}{q(t y) P(t)}, \lambda_{2} \sqrt{y}\right)-\Psi_{t y}\left(\lambda_{1}, \lambda_{2} \sqrt{y}\right) \\
& \quad=[q(t y)]^{-1} \underline{\Delta_{2}\left(t y ; \lambda_{1} \frac{q(t) P(t y)}{q(t y) P(t)}, \lambda_{2} \sqrt{y}\right)-\Delta_{2}\left(t y ; \lambda_{1}, \lambda_{2} \sqrt{y}\right)}
\end{aligned}
$$

Так как

$$
\Delta_{2}\left(u ; \lambda_{1}, \lambda_{2}\right)=\mathbf{E}\left[\exp \left\{-\lambda_{1} Z_{1}(u) \frac{q(u)}{P(u)}\right\}\left(1-\exp \left\{-Z_{2}(u) \frac{\lambda_{2}}{\sqrt{u}}\right\}\right)\right]
$$

то справедливо неравенство

$$
\begin{aligned}
J & \stackrel{\text { def }}{=}\left|\Delta_{2}\left(t y ; \lambda_{1} \frac{q(t) P(u)}{q(u) P(t)}, \lambda_{2} \sqrt{y}\right)-\Delta_{2}\left(t y ; \lambda_{1}, \lambda_{2} \sqrt{y}\right)\right| \\
& \leqslant \mathbf{E}\left[\left|\exp \left\{-\lambda_{1} Z_{1}(t y) \frac{q(t y)}{P(t y)}\right\}-\exp \left\{-\lambda_{1} Z_{1}(t y) \frac{q(t)}{P(t)}\right\}\right| ; Z_{1}(t y)>0\right] .
\end{aligned}
$$

Отметим, что, согласно теореме 1 и лемме 1 ,

$$
\frac{q(t y)}{P(t y)}=\frac{q(t)}{P(t)}(1+\gamma(t ; y))
$$

где $\gamma(t ; y) \rightarrow 0, t \rightarrow \infty$, равномерно по $y \in(\varepsilon, 1]$ для любого фиксированного $\varepsilon \in(0,1)$, и существует функция $\gamma(t) \in \mathscr{U}_{t}$ такая, что

$$
r(t) \stackrel{\text { def }}{=} \sup _{y \in(\varepsilon, 1]} \frac{q(t y)}{P(t y)}=\frac{q(t)}{P(t)}(1+\gamma(t)) .
$$

В силу неравенства треугольника для любых $a>0$ и $y \in(\varepsilon, 1]$

$$
\begin{aligned}
\left|\exp \left\{-a \frac{q(t y)}{P(t y)}\right\}-\exp \left\{-a \frac{q(t)}{P(t)}\right\}\right| \leqslant & \exp \left\{-a \frac{q(t y)}{P(t y)}\right\}-\exp \{-\operatorname{ar}(t)\} \\
& +\exp \left\{-a \frac{q(t)}{P(t)}\right\}-\exp \{-\operatorname{ar}(t)\} .
\end{aligned}
$$

Временно будем использовать символ $r^{*}(t)$ для обозначения любой из функций $r(t), \frac{q(t)}{P(t)}$ или $\frac{q(t y)}{P(t y)}$. Очевидно, что эти три функции асимптотически эквивалентны. Как следует из теоремы 2 , при $t \rightarrow \infty$ величина

$$
\begin{aligned}
& \mathbf{E}\left[\exp \left\{-\lambda_{1} Z_{1}(t y) r^{*}(t)\right\} ; Z_{1}(t y)>0\right] \\
& \quad=\mathbf{E}\left[\exp \left\{-\lambda_{1} Z_{1}(t y) r^{*}(t)\right\} \mid Z_{1}(t y)>0\right] q(t y)
\end{aligned}
$$

эквивалентна дроби $q(t y) /\left(1+\lambda_{1}\right)$ равномерно по $\lambda_{1} \in \mathscr{K}$ и равномерно по $y \in(\varepsilon, 1]$. Последнее влечет соотношение $J=o(q(t y)), t \rightarrow \infty$, что и завершает доказательство леммы. 
Лемма 9. При выполнении условий теоремь 3

$$
\begin{aligned}
& \frac{I_{2}\left(t ; 0, s_{2}(t)\right)-I_{2}\left(t ; s_{1}(t), s_{2}(t)\right)}{q(t)} \\
& \quad=-\alpha \sigma^{2} \lambda_{2} \int_{\varepsilon}^{1-\varepsilon} \Psi_{t y}\left(\lambda_{1}, \lambda_{2} \sqrt{y}\right) \frac{\varphi\left(\lambda_{2}^{2} y\right)}{\sqrt{y(1-y)}} d y+r_{\varepsilon}\left(t ; \lambda_{1}, \lambda_{2}\right),
\end{aligned}
$$

где

$$
\limsup _{\varepsilon \rightarrow+0} \limsup _{t \rightarrow \infty} \sup _{\left(\lambda_{1}, \lambda_{2}\right) \in \mathscr{K}^{2}}\left|r_{\varepsilon}\left(t ; \lambda_{1}, \lambda_{2}\right)\right|=0 .
$$

Д о к а з а т е л ь с т в о. Определения (34), (18) и (20) позволяют записать

$$
\begin{aligned}
& I_{2}\left(t ; 0, s_{2}(t)\right)-I_{2}\left(t ; s_{1}(t), s_{2}(t)\right) \\
& =\frac{\alpha \sigma^{2}}{2} \int_{0}^{t}\left(\Delta_{1}^{2}\left(t-u, t ; \lambda_{2}\right)-\Delta_{2}^{2}\left(t-u, t ; \lambda_{1}, \lambda_{2}\right)\right) P(u) d u \\
& +\alpha \int_{0}^{t} \frac{\sigma^{2}-h^{\prime \prime}\left(\theta\left(t-u ; s_{1}(t), s_{2}(t)\right)\right)}{2} \Delta_{2}^{2}\left(t-u, t ; \lambda_{1}, \lambda_{2}\right) P(u) d u \\
& -\alpha \int_{0}^{t} \frac{\sigma^{2}-h^{\prime \prime}\left(\theta\left(t-u ; 0, s_{2}(t)\right)\right)}{2} \Delta_{1}^{2}\left(t-u, t ; \lambda_{2}\right) P(u) d u \\
& \stackrel{\text { def }}{=} \frac{\alpha \sigma^{2}}{2} I_{3}+\alpha I_{3,1}-\alpha I_{3,2} \text {. }
\end{aligned}
$$

В силу (14), теоремы 1 и свойств промежуточного значения $\theta(*)$ из (35)

$$
\begin{aligned}
0 & \leqslant \max \left\{\theta\left(u ; s_{1}(t), s_{2}(t)\right), \theta\left(u, 0, s_{2}(t)\right)\right\} \leqslant Q\left(u ; 0, s_{2}(t)\right) \\
& \leqslant q(u)+1-s_{2}(t) \leqslant C_{1}(u+1)^{-1 / 2} \ln ^{-1}(u+2)+C_{2}(t+1)^{-1 / 2} .
\end{aligned}
$$

Отсюда, используя (5), несложно заключить, что

$$
0 \leqslant \sigma^{2}-h^{\prime \prime}\left(\theta\left(u ; s_{1}(t), s_{2}(t)\right)\right)=l_{1}(u) \ln ^{-1}(u+2)
$$

и

$$
0 \leqslant \sigma^{2}-h^{\prime \prime}\left(\theta\left(u ; 0, s_{2}(t)\right)\right)=l_{2}(u) \ln ^{-1}(u+2),
$$

где $l_{i}(u) \in \mathscr{U}_{t}, i=1,2$. Последние оценки и леммы 1 и 2 влекут при любом $\varepsilon \in\left(0, \frac{1}{2}\right)$ и $i=1,2$

$$
\begin{aligned}
0 \leqslant & I_{3, i} \leqslant \int_{0}^{t} l_{i}(t-u) \ln ^{-1}(t-u+2)\left(1-s_{2}(t)\right)^{2} P(u) d u \\
\leqslant & \frac{C_{1}}{(t+1) \sqrt{t+1} \ln (t+2)} \int_{t \varepsilon}^{t(1-\varepsilon)} l_{i}(t-u) d u \\
& +\frac{C_{2}}{(t+1) \sqrt{t+1}} \int_{t(1-\varepsilon)}^{t} \frac{d u}{\ln (t-u+2)}+\frac{C_{3}}{(t+1) \ln (t+2)} \int_{0}^{t \varepsilon} P(u) d u \\
= & o\left(\frac{1}{\sqrt{t+1} \ln (t+2)}\right)+O(1) \frac{\sqrt{\varepsilon}}{\sqrt{t+1} \ln (t+2)} .
\end{aligned}
$$


Итак,

$$
\limsup _{\varepsilon \rightarrow+0} \limsup _{t \rightarrow \infty} \sqrt{t} \ln t \sup _{\left(\lambda_{1}, \lambda_{2}\right) \in[0, \infty) \times[0, \infty)}\left(I_{3,1}+I_{3,2}\right)=0 .
$$

Интеграл $I_{3}$ в (52) можно записать в виде

$$
I_{3}=-\int_{0}^{t} \Delta\left(t-u, t ; \lambda_{1}, \lambda_{2}\right)\left(\Delta_{1}\left(t-u, t ; \lambda_{2}\right)+\Delta_{2}\left(t-u, t ; \lambda_{1}, \lambda_{2}\right)\right) P(u) d u \text {. }
$$

Проведем исследование асимптотического поведения данного интеграла при $t \rightarrow \infty$ отдельно в каждом из трех промежутков: $[0, t \varepsilon),[t \varepsilon, t(1-\varepsilon))$ и $[t(1-\varepsilon), t]$, где $\varepsilon \in\left(0, \frac{1}{2}\right)$ произвольное фиксированное.

Заметим, что в силу (23), леммы 2 и теоремы 1

$$
0 \leqslant \Delta\left(u, t ; \lambda_{1}, \lambda_{2}\right) \leqslant q(u ; 0)-q\left(u ; s_{1}(t)\right) \leqslant q(u ; 0) \leqslant \frac{C}{\sqrt{u+1} \ln (u+2)} .
$$

Отсюда с помощью лемм 1 и 2 легко вывести, что

$$
\begin{aligned}
I_{\varepsilon, 3}(t) \stackrel{\text { def }}{=} \int_{t(1-\varepsilon)}^{t} \Delta\left(t-u, t ; \lambda_{1}, \lambda_{2}\right) \\
\quad \times\left(\Delta_{1}\left(t-u, t ; \lambda_{2}\right)+\Delta_{2}\left(t-u, t ; \lambda_{1}, \lambda_{2}\right)\right) P(u) d u \\
\leqslant \frac{C_{1}}{\sqrt{t+1}} \int_{0}^{t \varepsilon} \Delta\left(u, t ; \lambda_{1}, \lambda_{2}\right)\left(\Delta_{1}\left(u, t ; \lambda_{2}\right)+\Delta_{2}\left(u, t ; \lambda_{1}, \lambda_{2}\right)\right) d u \\
\leqslant \frac{C_{2}\left(1-s_{2}(t)\right)}{\sqrt{t+1}} \int_{0}^{t \varepsilon} \frac{d u}{\sqrt{u+1} \ln (u+2)} \leqslant \frac{C \lambda_{2}}{t+1} \frac{\sqrt{\varepsilon t}}{\ln (\varepsilon t+2)}
\end{aligned}
$$

и, следовательно, для любого $\mathscr{K}^{2}$

$$
\limsup _{\varepsilon \rightarrow+0} \limsup _{t \rightarrow \infty} \sqrt{t} \ln t \sup _{\left(\lambda_{1}, \lambda_{2}\right) \in \mathscr{K}^{2}} I_{\varepsilon, 3}(t)=0 .
$$

Аналогичные аргументы показывают, что

$$
\begin{aligned}
I_{\varepsilon, 4}(t) \stackrel{\text { def }}{=} \int_{0}^{t \varepsilon} & \Delta\left(t-u, t ; \lambda_{1}, \lambda_{2}\right) \\
& \times\left(\Delta_{1}\left(t-u, t ; \lambda_{2}\right)+\Delta_{2}\left(t-u, t ; \lambda_{1}, \lambda_{2}\right)\right) P(u) d u \\
\leqslant & \frac{C_{1}}{(t+1) \ln (t+2)} \int_{0}^{t \varepsilon} P(u) d u \leqslant \frac{C}{(t+1) \ln (t+2)} \sqrt{\varepsilon t}
\end{aligned}
$$

и, следовательно, как и в (54),

$$
\lim _{\varepsilon \rightarrow+0} \limsup _{t \rightarrow \infty} \sqrt{t} \ln t \sup _{\left(\lambda_{1}, \lambda_{2}\right) \in \mathscr{K}^{2}} I_{\varepsilon, 4}(t)=0 .
$$

При помощи аргументов, сходных с теми, которые были использованы ранее при доказательстве данной леммы, можно также показать, 
привлекая леммы 6 и 8 , ограниченность $\lambda_{2} \varphi\left(\lambda_{2}^{2} y\right)$, равенство (28) и замечание 2, что

$$
\begin{aligned}
I_{\varepsilon, 5}(t) \stackrel{\text { def }}{=} & \int_{t \varepsilon}^{t(1-\varepsilon)} \Delta\left(t-u, t ; \lambda_{1}, \lambda_{2}\right) \Delta_{1}\left(t-u, t ; \lambda_{2}\right) P(u) d u \\
= & t \int_{\varepsilon}^{1-\varepsilon} \Delta\left(t y, t ; \lambda_{1}, \lambda_{2}\right) \Delta_{1}\left(t y, t ; \lambda_{2}\right) P(t(1-y)) d y \\
= & t \int_{\varepsilon}^{1-\varepsilon} q(t y) \Psi_{t y}\left(\lambda_{1} \frac{q(t) P(u)}{q(u) P(t)}, \lambda_{2} \sqrt{y}\right) \Delta_{1}\left(t y, t ; \lambda_{2}\right) P(t(1-y)) d y \\
= & t\left(1-s_{2}(t ; 1)\right) P(t) q(t) \\
& \times\left[\int_{\varepsilon}^{1-\varepsilon} \frac{\Psi_{t y}\left(\lambda_{1}, \lambda_{2} \sqrt{y}\right) \lambda_{2} \varphi\left(\lambda_{2}^{2} y\right)}{\sqrt{y(1-y)}} d y+\gamma_{1}\left(t ; \lambda_{1}, \lambda_{2}\right)\right] \\
= & {\left[\lambda_{2} \int_{\varepsilon}^{1-\varepsilon} \frac{\Psi_{t y}\left(\lambda_{1}, \lambda_{2} \sqrt{y}\right) \varphi\left(\lambda_{2}^{2} y\right)}{\sqrt{y(1-y)}} d y+\gamma_{2}\left(t ; \lambda_{1}, \lambda_{2}\right)\right] q(t) }
\end{aligned}
$$

где $\gamma_{i}\left(t ; \lambda_{1}, \lambda_{2}\right) \in \mathscr{U}_{t}, i=1,2$, и, кроме того,

$$
\begin{aligned}
I_{\varepsilon, 6}(t) & \stackrel{\text { def }}{=} \int_{t \varepsilon}^{t(1-\varepsilon)} \Delta\left(t-u, t ; \lambda_{1}, \lambda_{2}\right) \Delta_{2}\left(t-u, t ; \lambda_{1}, \lambda_{2}\right) P(u) d u \\
& =t \int_{\varepsilon}^{1-\varepsilon)} \Delta\left(t y, t ; \lambda_{1}, \lambda_{2}\right) \Delta_{2}\left(t y, t ; \lambda_{1}, \lambda_{2}\right) P(t(1-y)) d y \\
& =\left[\lambda_{2} \int_{\varepsilon}^{1-\varepsilon} \frac{\Psi_{t y}\left(\lambda_{1}, \lambda_{2} \sqrt{y}\right) \varphi\left(\lambda_{2}^{2} y\right)}{\sqrt{y(1-y)}} d y+\gamma_{1}\left(t ; \lambda_{1}, \lambda_{2}\right)\right] q(t),
\end{aligned}
$$

где $\gamma_{1}\left(t ; \lambda_{1}, \lambda_{2}\right) \in \mathscr{U}_{t}$. Итак,

$$
\frac{I_{\varepsilon, 5}+I_{\varepsilon, 6}}{q(t)}=2 \lambda_{2} \int_{\varepsilon}^{1-\varepsilon} \Psi_{t y}\left(\lambda_{1}, \lambda_{2} \sqrt{y}\right) \frac{\varphi\left(\lambda_{2}^{2} y\right)}{\sqrt{y(1-y)}} d y+\gamma\left(t ; \lambda_{1}, \lambda_{2}\right),
$$

где $\gamma\left(t ; \lambda_{1}, \lambda_{2}\right) \in \mathscr{U}_{t}$.

Используя в представлении (52) оценки (53)-(56), несложно завершить доказательство леммы 9.

Из равенств (31) и (32) и лемм 7 и 9 вытекает следующее утверждение.

Следствие 2. При выполнении условий теоремь 3

$$
\begin{aligned}
\Psi_{t}\left(\lambda_{1}, \lambda_{2}\right)= & \alpha \sigma^{2} \frac{\lambda_{2}}{1+\lambda_{1}} \int_{0}^{1} \frac{\varphi\left(\lambda_{2}^{2} y\right)}{\sqrt{y(1-y)}} d y \\
& -\alpha \sigma^{2} \lambda_{2} \int_{\varepsilon}^{1-\varepsilon} \frac{\Psi_{t y}\left(\lambda_{1}, \lambda_{2} \sqrt{y}\right) \varphi\left(\lambda_{2}^{2} y\right)}{\sqrt{y(1-y)}} d y+r_{1, \varepsilon}\left(t ; \lambda_{1}, \lambda_{2}\right),
\end{aligned}
$$

или, с учетом соотношений (25) и (27),

$$
\begin{aligned}
H_{t}\left(\lambda_{1}, \lambda_{2}\right)= & \frac{1}{1+\lambda_{1}}-\alpha \sigma^{2} \lambda_{2} \int_{\varepsilon}^{1-\varepsilon} \frac{H_{t y}\left(\lambda_{1}, \lambda_{2} \sqrt{y}\right) \varphi\left(\lambda_{2}^{2} y\right)}{\sqrt{y(1-y)}} d y \\
& +r_{2, \varepsilon}\left(t ; \lambda_{1}, \lambda_{2}\right)
\end{aligned}
$$


где

$$
\limsup _{\varepsilon \rightarrow+0} \limsup _{t \rightarrow \infty} \sup _{\left(\lambda_{1}, \lambda_{2}\right) \in \mathscr{K}^{2}}\left|r_{i, \varepsilon}\left(t ; \lambda_{1}, \lambda_{2}\right)\right|=0, \quad i=1,2 .
$$

Пусть, как и прежде, $\rho=\left(2 \alpha \pi \sigma^{2}\right)^{-1}$.

Лемма 10. В условиях теоремь 3 при всех $\left(\lambda_{1}, \lambda_{2}\right) \in[0, \infty) \times[0, \rho]$

$$
\lim _{t \rightarrow \infty} H_{t}\left(\lambda_{1}, \lambda_{2}\right)=\frac{D\left(\lambda_{2}\right)}{1+\lambda_{1}}
$$

Д о к а за т ель с тво. Легко убедиться в том, что функция $H\left(\lambda_{1}, \lambda_{2}\right) \stackrel{\text { def }}{=} D\left(\lambda_{2}\right)\left(1+\lambda_{1}\right)^{-1}$ в интересующей нас области изменения параметров $\lambda_{1}, \lambda_{2}$ удовлетворяет соотношению

$$
H\left(\lambda_{1}, \lambda_{2}\right)=\frac{1}{1+\lambda_{1}}-\alpha \sigma^{2} \lambda_{2} \int_{0}^{1} \frac{H\left(\lambda_{1}, \lambda_{2} \sqrt{y}\right) \varphi\left(\lambda_{2}^{2} y\right)}{\sqrt{y(1-y)}} d y .
$$

Более того, применяя рассуждения, использованные в лемме 3 при доказательстве существования и единственности решения уравнения (4), можно показать, что не существует функции $H\left(\lambda_{1}, \lambda_{2}\right)$, отличной от $D\left(\lambda_{2}\right)\left(1+\lambda_{1}\right)^{-1}$, которая удовлетворяла бы уравнению (58) на множестве $\left(\lambda_{1}, \lambda_{2}\right) \in[0, \infty) \times[0, \rho]$. Отсюда и из $(57)$ нетрудно вывести, что при любом $\varepsilon \in\left(0, \frac{1}{2}\right)$

$$
\begin{aligned}
& \left|H_{t}\left(\lambda_{1}, \lambda_{2}\right)-H\left(\lambda_{1}, \lambda_{2}\right)\right| \\
& \quad \leqslant \alpha \sigma^{2} \lambda_{2} \int_{\varepsilon}^{1-\varepsilon}\left|H_{t y}\left(\lambda_{1}, \lambda_{2} \sqrt{y}\right)-H\left(\lambda_{1}, \lambda_{2} \sqrt{y}\right)\right| \frac{\varphi\left(\lambda_{2}^{2} y\right)}{\sqrt{y(1-y)}} d y+r_{\varepsilon}\left(t ; \lambda_{1}, \lambda_{2}\right),
\end{aligned}
$$

где $\lim \sup _{\varepsilon \rightarrow+0} \lim \sup _{t \rightarrow \infty} \sup _{\left(\lambda_{1}, \lambda_{2}\right) \in \mathscr{K}^{2}}\left|r_{\varepsilon}\left(t ; \lambda_{1}, \lambda_{2}\right)\right|=0$ для любого $\mathscr{K}^{2} \subseteq[0, \infty) \times[0, \rho]$.

Полагая

$$
M_{t}\left(\lambda_{1} ; \lambda_{2}\right)=\sup _{0 \leqslant z \leqslant \lambda_{2}}\left|H_{t}\left(\lambda_{1}, z\right)-H\left(\lambda_{1}, z\right)\right|, \quad \mathscr{M}_{T}\left(\lambda_{1} ; \rho\right)=\sup _{t \geqslant T} M_{t}\left(\lambda_{1} ; \rho\right)
$$

и вспоминая (28), легко убедиться в том, что для достаточно больших $t$ и всех $\lambda_{1} \geqslant 0$ и $\lambda_{2} \in[0, \rho]$

$$
\left|H_{t}\left(\lambda_{1}, \lambda_{2}\right)-H\left(\lambda_{1}, \lambda_{2}\right)\right| \leqslant g(\varepsilon)+2^{-1} \sup _{y \geqslant \varepsilon} M_{t y}\left(\lambda_{1} ; \rho\right)=g(\varepsilon)+2^{-1} \mathscr{M}_{t \varepsilon}\left(\lambda_{1} ; \rho\right),
$$

где функция $g(\varepsilon)$ такова, что $\lim _{\varepsilon \downarrow 0} g(\varepsilon)=0$. Следовательно, при $t \geqslant t_{0}(\varepsilon)$

$$
M_{t}\left(\lambda_{1} ; \lambda_{2}\right) \leqslant g(\varepsilon)+2^{-1} \mathscr{M}_{t \varepsilon}\left(\lambda_{1} ; \rho\right) .
$$

Поэтому для всех достаточно больших $T$

$$
\mathscr{M}_{T}\left(\lambda_{1} ; \rho\right) \leqslant g(\varepsilon)+2^{-1} \mathscr{M}_{T \varepsilon}\left(\lambda_{1} ; \rho\right) .
$$

Вводя обозначение $\mathscr{M}\left(\lambda_{1} ; \rho\right)=\lim _{T \rightarrow \infty} \mathscr{M}_{T}\left(\lambda_{1} ; \rho\right)$, мы видим, что

$$
\mathscr{M}\left(\lambda_{1} ; \rho\right) \leqslant g(\varepsilon)+2^{-1} \mathscr{M}\left(\lambda_{1} ; \rho\right) .
$$


Поскольку $\varepsilon>0$ можно выбрать сколь угодно малым, это означает, что $\mathscr{M}\left(\lambda_{1} ; \rho\right)=0$.

Лемма доказана.

Вернемся к доказательству теоремы 3. Лемма 10, соотношения (25) и (10) и теорема единственности для преобразований Лапласа позволяют сделать вывод о сушествовании функции $W\left(\lambda_{1}, \lambda_{2}\right)$, аналитической в области $\operatorname{Re} \lambda_{1}>0, \operatorname{Re} \lambda_{2}>0$ и такой, что предел

$$
\lim _{t \rightarrow \infty} \mathbf{E}\left\{\exp \left\{-\frac{\lambda_{1} \zeta(t)}{\mathbf{E}[\zeta(t) \mid \zeta(t)>0]}-\frac{\lambda_{2} \mu(t)}{c_{p} \sqrt{t}}\right\} \mid \zeta(t)>0\right\}=W\left(\lambda_{1}, \lambda_{2}\right)
$$

существует для всех $\lambda_{1}, \lambda_{2}$, удовлетворяющих условию $\operatorname{Re} \lambda_{1}>0$, $\operatorname{Re} \lambda_{2}>0$. Более того,

$$
W\left(\lambda_{1}, \lambda_{2}\right)=H\left(\lambda_{1}, \lambda_{2}\right)=\frac{D\left(\lambda_{2}\right)}{1+\lambda_{1}} \quad \text { при } \quad \lambda_{1}, \lambda_{2} \in[0, \infty) \times[0, \rho] .
$$

Отсюда, полагая $\lambda_{1}=0$, заключаем, что существует и притом единственное аналитическое продолжение функции $D\left(\lambda_{2}\right)$ на область $\operatorname{Re} \lambda_{2}>0$. Мы сохраним для продолженной функции прежнее обозначение $D\left(\lambda_{2}\right)$. Ясно, что $D\left(\lambda_{2}\right)$ является на полупрямой $\lambda_{2}>0$ преобразованием Лапласа некоторого невырожденного распределения и, следовательно, не возрастает и положительна при $\lambda_{2}>0$. Этот факт в свою очередь означает, что

$$
W\left(\lambda_{1}, \lambda_{2}\right)=\lim _{t \rightarrow \infty} H_{t}\left(\lambda_{1}, \lambda_{2}\right)=\frac{D\left(\lambda_{2}\right)}{1+\lambda_{1}}, \quad \lambda_{1}, \lambda_{2} \in[0, \infty) \times[0, \infty),
$$

и для завершения доказательства теоремы достаточно проверить, что $D\left(\lambda_{2}\right)$ является решением уравнения (4) на всей полупрямой. Напомним, что единственность такого решения (если оно существует) была доказана в лемме 3. Оценка (15) и определение (25) влекут при $\lambda_{1}, \lambda_{2}>0$ соотношение $0 \leqslant H_{t}\left(\lambda_{1}, \lambda_{2}\right) \leqslant 1$, которое, ввиду неравенства $0 \leqslant \varphi(x) \leqslant 1$, $x \geqslant 0$, дает

$$
0 \leqslant \frac{H_{t}\left(\lambda_{1}, \lambda_{2}\right) \varphi\left(\lambda_{2}^{2} y\right)}{\sqrt{y(1-y)}} \leqslant \frac{1}{\sqrt{y(1-y)}} \quad \text { при всех } y \in(0,1), \lambda_{2} \geqslant 0 .
$$

Применяя к представлению (57) теорему Лебега о мажорируемой сходимости, получаем

$$
D\left(\lambda_{2}\right)=1-\alpha \sigma^{2} \lambda_{2} \int_{\varepsilon}^{1-\varepsilon} \frac{D\left(\lambda_{2} \sqrt{y}\right) \varphi\left(\lambda_{2}^{2} y\right)}{\sqrt{y(1-y)}} d y+r_{0, \varepsilon}\left(\lambda_{1}, \lambda_{2}\right),
$$

где $\lim \sup _{\varepsilon \rightarrow+0} \sup _{\left(\lambda_{1}, \lambda_{2}\right) \in \mathscr{K}^{2}}\left|r_{0, \varepsilon}\left(\lambda_{1}, \lambda_{2}\right)\right|=0$. Поскольку $0 \leqslant D\left(\lambda_{2}\right) \leqslant 1$ при любом $\lambda_{2}>0$, мы можем применить к последнему представлению теорему Лебега о мажорируемой сходимости при $\varepsilon \downarrow 0$ и получить, что $D\left(\lambda_{2}\right)$ является решением уравнения (4) на всей полупрямой.

Доказательство теоремы 3 завершено. 


\section{СПИСОК ЛИТЕРАТУРЫ}

1. Topchii V.A., Vatutin V.A., Yarovaya E. B. Catalytic branching random walk and queueing systems with random number of independent servers. - Theory Probab. Math. Statist., 2003, v. 69, p. 158-172.

2. Topchii V.A., Vatutin V.A. Individuals at the origin in the critical catalytic branching random walk. - Discrete Math. Theoret. Comput. Sci. (electronic), 2003, v. 6, p. 325332; http://dmtcs.loria.fr/proceedings/html/dmAC0130.abs.html

3. Ватутин B. A. Критические ветвящиеся процессы Беллмана-Харриса, начинающиеся с большого числа частиц. - Матем. заметки, 1986, т. 40, № 4, p. 527-541.

4. Albeverio $S$., Bogachev L. V. Branching random walk in a catalytic medium. I: Basic equations. - Positivity, 2000, v. 4, № 1, p. 41-100.

5. Albeverio S., Bogachev L. V., Yarovaya E.B. Asymptotics of branching symmetric random walk on the lattice with a single source. C. R. Acad. Sci. Paris, 1998, v. 326, № 8, p. 975-980; Corrections: ibid., v. 327, №6, p. 585.

6. Богачев Л.В., Яровая Е.Б. Предельная теорема для надкритического ветвящегося случайного блуждания на $\mathbf{Z}^{d}$ с одним источником. - Успехи матем. наук, 1998, т. 53 , в. 5 , с. $1086-1088$.

7. Богачев Л. В., Яровая Е. Б. Моментный анализ ветвящегося случайного блуждания на решетке с одним источником. - Докл. РАН, 1998, т. 363, № 4, с. 439-442.

8. Fleischmann K. Superprocesses in catalytic media. - Measure-valued Processes, Stochastic Partial Differential Equations, and Interacting Systems (Montréal, 1992). Ed. by D. A. Dawson et al., Providence, RI: Amer. Math. Soc., 1994, p. 99-110. (CRM Proc. Lecture Notes, v. 5,)

9. Fleischmann K., Le Gall J.-F. A new approach to the single point catalytic superBrownian motion. - Probab. Theory Related Fields, 1995, v. 102, № 1, p. 63-82.

10. Севастьянов Б. А. Ветвящиеся процессы. М.: Наука, 1971, 436 с.

11. Харрис T. Теория ветвящихся случайных процессов. М.: Мир, 1966, 355 с.

12. Ватутин В.А. Дискретные предельные распределения числа частиц в ветвящихся процессах Беллмана-Харриса с несколькими типами частиц. - Теория вероятн. и ее примен., 1979, т. 24, в. 3, с. 503-514.

13. Vatutin $V$., Xiong J. Some limit theorems for a particle system of single point catalytic branching random walks. - Acta Mathematica Sinica (в печати).

Поступила в редакцию 19.I.2004 